\title{
Probiotic and synbiotic therapy in critical illness: a systematic review and meta-analysis
}

\author{
William Manzanares ${ }^{1}$, Margot Lemieux², Pascal L. Langlois ${ }^{3}$ and Paul E. Wischmeyer ${ }^{4^{*}}$
}

\begin{abstract}
Background: Critical illness is characterized by a loss of commensal flora and an overgrowth of potentially pathogenic bacteria, leading to a high susceptibility to nosocomial infections. Probiotics are living non-pathogenic microorganisms, which may protect the gut barrier, attenuate pathogen overgrowth, decrease bacterial translocation and prevent infection. The purpose of this updated systematic review is to evaluate the overall efficacy of probiotics and synbiotic mixtures on clinical outcomes in critical illness.

Methods: Computerized databases from 1980 to 2016 were searched. Randomized controlled trials (RCT) evaluating clinical outcomes associated with probiotic therapy as a single strategy or in combination with prebiotic fiber (synbiotics). Overall number of new infections was the primary outcome; secondary outcomes included mortality, ICU and hospital length of stay (LOS), and diarrhea. Subgroup analyses were performed to elucidate the role of other key factors such as probiotic type and patient mortality risk on the effect of probiotics on outcomes.

Results: Thirty trials that enrolled 2972 patients were identified for analysis. Probiotics were associated with a significant reduction in infections (risk ratio $0.80,95 \%$ confidence interval $(C l) 0.68,0.95, P=0.009$; heterogeneity $P^{2}=36 \%, P=0.09$ ). Further, a significant reduction in the incidence of ventilator-associated pneumonia (VAP) was found (risk ratio $0.74,95 \% \mathrm{Cl} 0.61,0.90, P=0.002 ; P^{2}=19 \%$ ). No effect on mortality, LOS or diarrhea was observed. Subgroup analysis indicated that the greatest improvement in the outcome of infections was in critically ill patients receiving probiotics alone versus synbiotic mixtures, although limited synbiotic trial data currently exists.

Conclusion: Probiotics show promise in reducing infections, including VAP in critical illness. Currently, clinical heterogeneity and potential publication bias reduce strong clinical recommendations and indicate further high quality clinical trials are needed to conclusively prove these benefits.
\end{abstract}

Keywords: Probiotics, Synbiotics, Critical care, Infections, Ventilator-associated pneumonia, Systematic review

\section{Background}

Critical illness is characterized by a loss of commensal flora and an overgrowth of potentially pathogenic bacteria, leading to a high susceptibility to acquired nosocomial infections $[1,2]$. Further, sepsis following infection is still a leading cause of death worldwide [3]. The U.S. Centers for Disease Control indicates death rates from critical illness/sepsis have increased at a rate greater than any other common cause of mortality in the last year for

\footnotetext{
* Correspondence: Paul.Wischmeyer@ucdenver.edu

${ }^{4}$ Department of Anesthesiology and Pediatrics (Nutrition Section), University of Colorado, School of Medicine, 12700 E. 19th Ave., RC2 P15-7120, Box 8602, Aurora, CO 80045, USA

Full list of author information is available at the end of the article
}

which data were available [4]. Thus, therapies to reduce the risk and incidence of infection and sepsis in critical illness are urgently needed.

According to the World Health Organization and the Food and Agriculture Organization, probiotics are living non-pathogenic microorganisms, which have demonstrated well-documented beneficial health effects administered in optimum amounts in the prevention and treatment of several disease states [5]. So far, several mechanisms by which probiotics may exert beneficial effects have been described, including modification of the gut flora by inducing host cell antimicrobial peptides, release of antimicrobial factors, suppression of the 
immune cell proliferation, stimulation of mucus and IgA production, anti-oxidative activity, inhibition of epithelial cell nuclear factor kappa B activation, and other potentially vital gut epithelial barrier protective effects [6-8]. As the gut is hypothesized to play a central role in the progression of critical illness, sepsis and multiple organ dysfunction syndrome [9], maintenance of the gut barrier and a healthy gut microbiome, potentially via reintroduction of commensal bacteria (probiotic therapy), may be essential to optimizing outcomes in critically ill patients.

According to current literature, the efficacy of probiotics in the prevention of infectious complications has been extensively evaluated in many animal studies and clinical trials in heterogenous intensive care unit (ICU) patient populations. These studies suggest that probiotics may reduce the incidence of infection, particularly ventilator-associated pneumonia (VAP) [10], which is a common serious complication in intubated, mechanically ventilated patients [11]. Nonetheless, the effect of probiotics on the prevention of VAP still remains controversial and inconclusive [12-17]. In fact, its effect depends on the patient population and the probiotic strain studied. Despite the outcome benefits of probiotics therapy, recent guidelines have been unable to make a definitive recommendation for the routine use of probiotics in ICU patients. To date, these guidelines have suggested the use of probiotic therapy in select medical and surgical patient populations in whom trials have documented safety and clinical benefits [18, 19].

Over the last few years, several systematic reviews and meta-analyses have evaluated the effects of probiotics in critically ill patients [12-17]. In 2012, after aggregating 11 trials that reported on infections [14], we demonstrated that probiotics may reduce infections, including the incidence of VAP, although the effect on VAP was not statistically significant given the available data. Moreover, probiotics were associated with a trend toward reduced ICU mortality, but did not influence hospital mortality. Since our last systematic review and meta-analyses, seven new trials of probiotic therapy have been published [20-26]. Further, to date, no recent meta-analysis has examined the effect of probiotic versus synbiotic (probiotic and prebiotic fiber) therapy. Finally, a Canadian survey [27] on the use of probiotics as a prophylactic strategy for VAP showed that most Canadian ICU pharmacists have used probiotics at least once, although routine use is considered controversial and considerable practice variability exists. Thus, any increased understanding that the newly published trials can yield will be vital to clarifying clinical probiotic use in the ICU and areas in need of future research focus.

Therefore, as probiotic use in the ICU remains widespread and controversial, current guidelines are not conclusive, and with a significant number of new trials of probiotic use published recently we conducted a comprehensive systematic review and meta-analysis of probiotic and synbiotic use in critically ill patients. Our aim was to elucidate the overall efficacy of probiotics, as a single strategy or in combination with fiber therapy (synbiotics) on relevant clinical outcomes, particularly infection and VAP, in adult critically ill patients.

\section{Methods}

\section{Search strategy and study identification}

A literature search was conducted in MEDLINE, Embase, CINAHL, the Cochrane Central Register of Controlled Trials and the Cochrane Database of Systematic Reviews to identify all relevant randomized controlled trials (RCTs) published between 1980 and April 2016. The literature search used broad search terms containing "randomized," "clinical trial," "nutrition support," "enteral nutrition", "probiotics," and "synbiotics". No language restrictions were applied. Personal files and reference lists of relevant review articles were also reviewed.

\section{Eligibility criteria}

We included trials with the following characteristics:

1. Type of study: randomized controlled parallel group trials

2. Population: adult ( $\geq 18$ years of age) critically ill patients. If the study population was unclear, we considered a mortality rate higher than $5 \%$ in the control group to be consistent with critical illness

3. Intervention: Probiotics alone or associated with prebiotics (synbiotics) compared to a placebo

4. Outcomes: pre-specified clinical outcomes in ICU patients such as infectious complications, VAP, mortality, ICU and hospital length of stay (LOS), and diarrhea

We excluded trials that reported only nutrition, biochemical, metabolic, or immunologic outcomes. Data published in abstract form were included only if additional information about the study design was obtained from the authors. The methodological quality of the included trials was assessed in duplicate by two reviewers independently using a data abstraction form with a scoring system from 0 to 14 according to the following criteria:

1. The extent to which randomization was concealed

2. Blinding

3. Analysis based on the intention-to-treat (ITT) principle

4. Comparability of groups at baseline

5. Extent of follow up 
6. Description of treatment protocol

7. Co-interventions

8. Definition of clinical outcomes

Consensus between both reviewers on the individual scores of each of the categories was obtained. We attempted to contact the authors of included studies and requested additional information not contained in published articles. We designated studies as level I if all of the following criteria were fulfilled: concealed randomization, blinded outcome adjudication and an ITT analysis, all which are the strongest methodological tools to reduce bias. A study was considered as level II if any one of the above-described characteristics were unfulfilled.

\section{Data synthesis}

All analyses, except the test for asymmetry, were conducted using RevMan 5.3 (Cochrane IMS, Oxford, UK) with a random effects model. We combined data from all trials to estimate the overall weighted mean difference (WMD) with $95 \%$ confidence intervals for LOS data the pooled risk ratio (RR) with $95 \%$ confidence intervals (CIs) for the incidence of infections and mortality, and diarrhea. WMDs were estimated by the inverse variance approach and pooled RRs were calculated using the Mantel-Haenszel estimator. The random effects model of DerSimonian and Laird was used to estimate variances for the MantelHaenszel and inverse variance estimators [28]. RRs were undefined and excluded for studies with no event in either arm. Heterogeneity was tested by a weighted Mantel-Haenszel $X^{2}$ test and quantified by the $I^{2}$ statistic as implemented in RevMan. Differences between subgroups were analyzed using the test of subgroup differences described by Deeks et al., and the results expressed using the $P$ values. We considered $P<0.05$ to be statistically significant and $P<0.10$ as an indicator of trends. Funnel plots were used to assess the possibility of publication bias and the Egger regression test was used to measure funnel plot asymmetry [29].

\section{Clinical outcomes}

Overall infections were the primary outcome for this meta-analysis. Secondary outcomes were VAP, mortality, ICU and hospital LOS, and finally diarrhea. We used definitions of infections as defined by the authors in their original articles. From all trials, we combined hospital mortality where reported. Mortality specified at either 28 days or 90 days was not considered as ICU or hospital mortality, respectively. Nonetheless, if the mortality time frame was not specified as either ICU or hospital, it was presumed to be the later.

\section{Subgroup analysis}

We utilized predefined subgroup analyses to assess a number of possible influences on the effect of probiotic supplementation on clinical outcomes, and thus to explore the possible causes of heterogeneity. On the basis that the higher the daily dose the greater the effect, we first examined trials that administered a high dose of probiotics defined as $>5 \times 10^{9}$ colony-forming units $(\mathrm{CFU}) /$ day vs. lower dose probiotics defined as $<5 \times$ $10^{9} \mathrm{CFU} /$ day. Second, we compared the results of RCTs that administered Lactobacillus plantarum as probiotic therapy vs. no L. Plantarum, and compared trials using Lactobacillus rhamnosus strain GG (LGG) vs. those administering other non-LGG strains.

Moreover, based on a larger treatment effect in those more seriously ill patients with higher risk of death, we compared studies including patients with higher mortality vs. lower mortality. Mortality was considered to be high or low based on whether it was greater or less than the median control group mortality of all the trials. Trials of higher quality, defined as those with a methodological score equal to or higher than the median quality score, may demonstrate a lower treatment effect.

\section{Results}

\section{Study identification and selection}

A total of 79 relevant citations were identified from the search of computerized bibliographic databases and a review of reference lists from related articles. Of these, we excluded 49 due to the following reasons: 21 trials did not include ICU patients (mostly surgical patients); 12 articles were systematic reviews and meta-analyses; 4 trials were published as an abstract and we were unable to obtain the data from the authors to complete our data abstraction process; 5 articles were duplicates of included trials; 3 studies did not evaluate clinical outcomes; 2 trials tested multiple interventions; 1 study was not a RCT, and finally 1 study administered probiotics as oral swabs.

Finally, 30 RCTs [10, 20-26, 30-51] met our inclusion criteria and were included, covering a total of 2972 patients (see Tables 1 and 2). The reviewers reached $100 \%$ agreement on the inclusion of the trials. The mean methodological score of all trials was 9, whereas the median value was 9.5 on a maximum of 14 (range 5-13). Randomization was concealed in $9 / 30$ trials (30\%), ITT analysis was performed in 18/30 trials (60\%), and double blinding was done in $20 / 30$ of the studies (67\%). There were five level-I studies and 25 level-II studies. The details of the methodological quality of the individual trials are shown in Table 1.

\section{Primary outcome: infections Overall effect on new infections}

Aggregating the results of the 14 trials reporting overall infections, probiotics were associated with a significant 
Table 1 Randomized studies evaluating probiotics in critically ill patients
Study

$1 \quad$ Tempe 1983 [30]

ICU patients
$n=40$

2 Schlotterer 1987 [31]

Patients with burns $n=18$

$3 \quad$ Heimburger 1994 [32]

Mixed ICU patients: $83 \%$ received antibiotics $n=62$

$4 \quad$ Bleichner $1997[33]$

Mixed ICU patients $n=128$

$5 \quad$ Kecskes $2003[34]$

ICU patients on antibiotics $n=45$

6 Jain $2004[36]$

ICU patients

$n=90$

$7 \quad$ Lu 2004 [35]

8 Klarin 2005 [37]

C.Random: no
Patients with burns $n=40$

Critically ill patients on antibiotics $n=17$

ICU patients on antibiotics $n=130$
C.Random: yes

ITT: yes

Blinding: double

Score: 10

Viability (intervention): NR

C.Random: no

ITT: no

Blinding: double

Score: 8

Viability (intervention): NR

ITT: no

Blinding: double

Score: 9

Viability (intervention): NR

C.Random: not sure

ITT: yes

Blinding: double

Score: 13

Viability (intervention): NR

C.Random: no

ITT: no

Blinding: double

Score: 8

Viability (intervention): yes

C.Random: no

ITT: yes

Blinding: double

Blinding:

Viability (intervention): NR

C.Random: no

ITT: yes

Blinding: double

Score: 9

Viability (intervention): NR

C.Random: no

ITT: no

Blinding: no

Score: 6

Viability (intervention): NR

C.Random: no

ITT: yes

Blinding: no

Score: 7

Viability (intervention): NR
Type of probiotic/intervention

Delivery vehicle Intervention/dose/duration

EN (unknown) + Ultra-Levure

Control

EN tube

(Saccharomyces boultrardii)

EN (unknown) + placebo

$10^{10} / 1 \mathrm{~L}$ solution for $11-21$ days

(sterile solution)

NG tube

EN (Polydiet or Nutrigil) +

Saccharomyces boulardi

500 mg QID for 8-28 days

EN (Polydiet or Nutrigil) + placebo

EN (standard) $+1 \mathrm{~g}$ of Lactinex (Lactobacillus acidophilus and Lactobaccilus bulgaricus) $2 \times 10^{6}$ TID for 5-10 days

EN tube

EN (unknown) + Saccharomyces boulardii

$500 \mathrm{mg}$ QID for 21 days or

until EN stopped

NJ tube $\quad$ EN (Nutrison fiber) + fermented oatmeal formula with Lactobacillus plantarum $29910^{9}$ BID and fiber for 7 days

Oral or NG tube

EN or PN + Trevis 1 TM 1 capsule TID +

7.5 g Raftilose (oligofructose)

BID until hospital discharge

NR

EN + synbiotics (4 types of probiotics +4 types of unspecified prebiotics) for 21 days

Mixed in fermented oatmeal, given via NG tube

$\mathrm{EN}+$ Lactobacillus plantarum $299 \mathrm{~V}$, $10^{9} /$ day $50 \mathrm{ml}$ every $6 \mathrm{~h} \times 3$ days then $25 \mathrm{ml}$ every $6 \mathrm{~h}$ until ICU discharge

Oral, NJ tube

EN or PN + Proviva, (oatmeal and fruit drink) $5 \times 10^{7} \mathrm{CFU} / \mathrm{ml}$ of L. plantarum $299 \mathrm{v} \times 500 \mathrm{mls}$ until hospital discharge or beyond
EN (standard) + placebo

(0.5 g dextrose $+0.5 \mathrm{~g}$ lactose)

EN (unknown) + placebo (powder)

EN (Nutrison fiber) + heat-killed Lactobacillus plantarum 299 BID + fiber (non-viable)

$\mathrm{EN}$ or $\mathrm{PN}+$ placebo

(powdered sucrose capsules)

EN +4 types of prebiotics

EN (Impact or Nutrodrip fiber). Some patients needed PN

EN or PN alone 
Table 1 Randomized studies evaluating probiotics in critically ill patients (Continued)

10 Kotzampassi 2006 [39] Patients with multiple trauma from 5 ICUs $n=77$

11 Alberda $2007[40]$

ICU patients $n=28$ Li 2007 [41]

3 Olah 2007 [42]

14 Forestier $2008[44]$

15 Besselink $2008[43]$

16 Klarin $2008[45]$

17 Knight 2009 [46]

pancreatitis

$n=25$

Patients with sever

acute pancreatitis

$n=83$

Mixed ICU patients, $50 \%$ on antibiotics

$n=208$ pancreatitis from $15 \mathrm{ICUs}$ $n=298$

ICU patients from 5 ICUs,

$n=68$

General ICU patients

\section{Random: no}

ITT: no

Blinding: double

Score: 8

Viability (intervention): NR

VAP determination: clinica

C.Random: no

TT: yes;

Score: 10

Viability (intervention): No

for VSL \# 3; Yes for bacteria

sonicates

C.Random: no

ITT: yes

Patients with severe acute

Blinding: no

Score: 7

Viability (intervention): NR

C.Random: no

ITT: no

Blinding: no

Viability (intervention): NR

C.Random: not sure

ITT: no

Blinding: double

Score: 8

Viability (intervention): NR

VAP determination: objective

C.Random: not sure

Patients with severe acute

ITT: yes

Blinding: double

Score:11

Viability (intervention): NR

VAP determination: clinica

C.Random: yes

ITT: no on antibiotics for C. Difficile

Blinding: double

Score: 10

Viability (intervention): NR

$n=300$

ines

ITT: no
Blinding: double

Endoscopic

gastrostomy

or NG tube

EN or PN + Synbiotic 2000 Forte

111 PN + Synbiotic 2000 Forte

until ICU discharge

NG tube

evity Plus (EN) (10 g

(10)

and $12 \mathrm{~g}$ of soluble and

insoluble fiber blend) +

VSL \# 3, 1 package BID,

$9 \times 10^{11} /$ day for 7 days until

ICU discharge or EN

discontinuation

Given enterally

Jinshuangqi (bifidobacteria,

lactobacillus and streptococcus)

$2.0 \mathrm{~g}$ TID on basis of traditional

treatment

Duration: NR

NJ tube

EN (Nutricion fiber) + Synbiotic

$2000,4 \times 10^{10}$ CFU for 7 days

NG tube or oral

(after tube removal)

Lactobacillus casei rhamnosum, $10^{9}$ CFU BID until ICU discharge

NJ tube or ora

EN (Nutrison multifiber) + Ecologic $64110^{10}$ CFU BID for 28 days

299 Lactobacillus plantarum

Mixed in fermented oatmeal added to

enteral feeds NG tube $8 \times 10^{8} \mathrm{CFU} / \mathrm{ml}$ given as $6 \times 100 \mathrm{~m}$ doses every $12 \mathrm{~h}$ and after $50 \mathrm{ml}$ given BID until ICU discharge

\section{NJ or OG (orogastric) EN (Nutrition Energy) +}

Blinding: double

Viability (intervention): NR

VAP determination: clinica
Synbiotic 2000 FORTE

BID for 28 days or ICU discharge tube$$
\text { }
$$

EN or PN + placebo (maltodextrin)

mixed in tap water

Jevity Plus + placebo

Traditional treatment

EN (Nutricion fiber) +10 plant fibers ( $2.5 \mathrm{~g}$ each of Betaglucan, inulin, pectin and resistant starch) (prebiotics) BID for at least 2 days

Placebo (growth medium never exposed to bacteria)

EN (Nutrison multifiber) + placebo (cornstarch + maltodextrins)

Same oatmeal gruel mixed with lactic acid

EN (Nutrison Energy) + Placebo 
Table 1 Randomized studies evaluating probiotics in critically ill patients (Continued)

\begin{tabular}{|c|c|c|c|c|c|c|}
\hline 18 & Barraud 2010 [47] & $\begin{array}{l}\text { Mechanically ventilated ICU } \\
\text { patients, } 80 \% \text { on antibiotics } \\
n=167\end{array}$ & $\begin{array}{l}\text { C.Random: yes } \\
\text { ITT: yes; } \\
\text { Blinding: double } \\
\text { Score: } 12 \\
\text { Viability (intervention): NR } \\
\text { VAP determination: objective }\end{array}$ & NG tube & $\begin{array}{l}\text { EN (Fresubin) + Ergyphilus } \\
2 \times 10^{10} \text { per capsule + potato } \\
\text { starch } 5 \text { capsules/day for } 28 \text { days }\end{array}$ & $\begin{array}{l}\text { EN (fresubin) + placebo capsules } \\
\text { (excipient of potato starch) }\end{array}$ \\
\hline 19 & Morrow 2010 [10] & $\begin{array}{l}\text { ICU patients } \\
\mathrm{n}=146\end{array}$ & $\begin{array}{l}\text { C.Random: no; } \\
\text { ITT: yes; } \\
\text { Blinding: double; Score: } 10 \\
\text { Viability (intervention): yes } \\
\text { VAP determination: objective }\end{array}$ & $\begin{array}{l}\text { Oropharynx and } \\
\text { NG tube }\end{array}$ & $\begin{array}{l}\text { EN (routine care) + Lactobacillus } \\
\text { rhamnosus GG, } 2 \times 10^{9} \text { BID as } \\
\text { lubricant and mixed with water } \\
\text { until extubation }\end{array}$ & $\begin{array}{l}\text { EN (routine care) }+ \text { inert plant } \\
\text { starch inulin (prebiotic) BID as } \\
\text { lubricant and mixed with water }\end{array}$ \\
\hline 20 & Frohmader 2010 [48] & $\begin{array}{l}\text { General ICU patients } \\
\text { on antibiotics } \\
n=45\end{array}$ & $\begin{array}{l}\text { C.Random: yes } \\
\text { ITT: yes } \\
\text { Blinding: double } \\
\text { Score: } 11 \\
\text { Viability (intervention): yes }\end{array}$ & NG or NJ tube & $\begin{array}{l}\text { EN (Standard) + VSL \#3 mixed in } \\
\text { nutritional supplement (Sustagen), } \\
\text { BID until hospital discharge }\end{array}$ & $\begin{array}{l}\text { EN (Standard) + placebo } \\
\text { mixed in nutritional } \\
\text { supplement (Sustagen), BID }\end{array}$ \\
\hline 21 & Ferrie 2011 [49] & $\begin{array}{l}\text { Critically ill patients } \\
\text { with diarrhea, } \\
n=36\end{array}$ & $\begin{array}{l}\text { C.Random: no } \\
\text { ITT: yes } \\
\text { Blinding: double } \\
\text { Score: } 10 \\
\text { Viability (intervention): yes }\end{array}$ & NG tube & $\begin{array}{l}\text { EN (Standard) + Culturelle } \\
\text { (Lactobacillus rhamnosus } G G), \\
10^{10} \text { species/capsule } \\
+280 \mathrm{mg} \text { inulin powder for } 7 \text { days }\end{array}$ & $\begin{array}{l}\text { EN (Standard) + Raftiline, gelatin } \\
\text { capsule with } 280 \text { mg inulin } \\
\text { powder (prebiotic) }\end{array}$ \\
\hline 22 & Sharma 2011 [50] & $\begin{array}{l}\text { Patients with acute } \\
\text { pancreatitis } \\
n=50\end{array}$ & $\begin{array}{l}\text { C.Random: yes } \\
\text { ITT: yes } \\
\text { Blinding: double } \\
\text { Score: } 11 \\
\text { Viability (intervention): yes }\end{array}$ & Oral, NJ or NG & $\begin{array}{l}\text { EN (standard) or oral } \\
4 \text { sachets each } 2.5 \times 10^{9} \\
\text { Lactobacillus acidophilus, Bifidobacterium } \\
\text { longus, Bifidobacterium bifidum \& } \\
\text { Bifidobacterium infantalis }+25 \mathrm{gms} \\
\text { fructose for } 7 \text { days }\end{array}$ & EN (Standard) + placebo \\
\hline 23 & $\operatorname{Tan} 2011$ [51] & $\begin{array}{l}\text { Patients with closed } \\
\text { head injury } \\
n=52\end{array}$ & $\begin{array}{l}\text { C.Random: yes } \\
\text { ITT: yes } \\
\text { Blinding: single } \\
\text { Score: } 10 \\
\text { Viability (intervention): yes } \\
\text { VAP determination: clinical }\end{array}$ & NG tube & $\begin{array}{l}\text { EN (standard) } \\
\text { total of } 10^{9} \text { bacteria i.e., } \\
7 \text { sachets each } 0.5 \times 10^{8} \text { Bifidobacterium } \\
\text { longum, } 0.5 \times 10^{7} 1 \text { Lactobacillus } \\
\text { bulgaricus and } 0.5 \times 10^{7} \text { Streptococcus } \\
\text { thermophilus for } 21 \text { days }\end{array}$ & EN (standard) \\
\hline 24 & Cui 2013 [20] & $\begin{array}{l}\text { Patients with severe acute } \\
\text { pancreatitis } \\
\mathrm{n}=70\end{array}$ & $\begin{array}{l}\text { C.Random: no } \\
\text { ITT: yes } \\
\text { Blinding: no } \\
\text { Score: } 9 \\
\text { Viability (intervention): yes }\end{array}$ & EN & $\begin{array}{l}\text { EN }+ \text { bifidobacterium, } 4 \text { capsules } \\
\left(\text { each } 210 \mathrm{mg}, 2.604 \times 10^{9}\right) \text { every } \\
12 \mathrm{~h} \text {, given through nasal gastric } \\
\text { tube. Total dose per day } 20.832 \times 10^{9}\end{array}$ & EN \\
\hline 25 & Tan 2013 [21] & $\begin{array}{l}\text { Severe craniocerebral trauma } \\
n=52\end{array}$ & $\begin{array}{l}\text { C.Random: no } \\
\text { ITT: other } \\
\text { Blinding: no } \\
\text { Score: } 11 \\
\text { Viability (intervention): yes }\end{array}$ & NG tube & $\begin{array}{l}\text { EN }+1 \times 10^{9} \text { bacteria of viable } \\
\text { probiotics (Golden Bifid, } 3.5 \mathrm{~g} 3 \text { times } \\
\text { per day) per day for } 21 \text { days. }\end{array}$ & EN (standard) \\
\hline
\end{tabular}


Table 1 Randomized studies evaluating probiotics in critically ill patients (Continued)

\begin{tabular}{|c|c|c|c|c|c|c|}
\hline 26 & Wang 2013 [22] & $\begin{array}{l}\text { Severe acute pancreatitis } \\
\text { with intestinal ileus or } \\
\text { abdominal distention. } \\
n=183\end{array}$ & $\begin{array}{l}\text { C.Random: no } \\
\text { IT: yes } \\
\text { Blinding: no } \\
\text { Score: } 6 \\
\text { Viability (intervention): NR }\end{array}$ & SBFT & $\begin{array}{l}\text { EN (standard) + capsules } 0.5 \mathrm{~g} \text { TID } \\
\text { containing Bacillus subtilis and } \\
\text { Enterococcus faecium }\left(5.0 \times 10^{7}\right. \\
\text { Bacillus subtilis and } 4.5 \times 10^{8} \\
\text { Enterococcus faecium per } 250 \mathrm{~g} \\
\text { capsule). Unclear timeframe. }\end{array}$ & EN (standard) \\
\hline 27 & $\begin{array}{l}\text { Lopez de Toro } 2014 \\
\text { [23] }\end{array}$ & $\begin{array}{l}\text { Medical and surgical ICU } \\
\text { patients with multi-organ } \\
\text { failure } \\
n=89\end{array}$ & $\begin{array}{l}\text { C.Random: yes } \\
\text { ITT: yes } \\
\text { Blinding: no } \\
\text { Score: } 11 \\
\text { Viability (intervention): NR }\end{array}$ & EN & $\begin{array}{l}\text { EN + symbiotic drink with } \\
\text { streptococcus Thermophilus, } \\
\text { lactobacillus bulgaricus, Lactobacillus } \\
\text { casei, lactobacillus acidophilus, } \\
\text { bifidobacterium, Escherichia coli, } \\
\text { coliformes } \times 7 \text { days (max } 4.8 \times \\
10^{9} \mathrm{UFC} / \mathrm{ml} \text { ). }\end{array}$ & EN and PN \\
\hline 28 & Sanaie 2014 [24] & $\begin{array}{l}\text { Critically ill pts, SIRS, expected } \\
\text { length of stay } \geq 7 \text { days } \\
n=40\end{array}$ & $\begin{array}{l}\text { C.Random: yes } \\
\text { ITT: yes } \\
\text { Blinding: double } \\
\text { Score: } 9 \\
\text { Viability (intervention): yes }\end{array}$ & NG tube & $\begin{array}{l}\text { EN (standard) }+2 \text { sachets VSL\#3 } \\
\text { BID } \times 7 \text { days. }\end{array}$ & EN (standard) + placebo \\
\hline 29 & $\begin{array}{l}\text { Rongrungruang } 2015 \\
{[25]}\end{array}$ & $\begin{array}{l}\text { Critically ill patients, } \\
\text { expected to receive } \\
\text { mechanical } \\
\text { ventilation at least } 72 \mathrm{~h} \text { and } \\
\text { had no VAP at enrollment } \\
n=150\end{array}$ & $\begin{array}{l}\text { C.Random: no } \\
\text { ITT: no } \\
\text { Blinding: no } \\
\text { Score: } 6 \\
\text { Viability (intervention): yes }\end{array}$ & EN & $\begin{array}{l}80 \mathrm{ml} \text { of } 8 \times 10^{9} \text { cfu of Lactobacillus } \\
\text { casei (Shirota strain) (Yakult) for } \\
\text { oral care after the standard oral } \\
\text { care once daily } \\
\text { An additional } 80 \mathrm{ml} \text { of the product } \\
\text { was given via enteral feeding once } \\
\text { daily for } 28 \text { days or when their } \\
\text { endotracheal tubes were removed }\end{array}$ & $\begin{array}{l}\text { EN (standard) + oral care with } \\
2 \% \text { chlorhexidine solution } \\
4 \text { times per day }\end{array}$ \\
\hline 30 & Zeng 2016 [26] & $\begin{array}{l}\text { Critically ill patients, } \\
\text { expected to receive } \\
\text { mechanical } \\
\text { ventilation at least } 48 \mathrm{~h} \\
n=235\end{array}$ & $\begin{array}{l}\text { C.Random: yes } \\
\text { ITT: no } \\
\text { Blinding: no } \\
\text { Score: } 5 \\
\text { Viability (intervention): yes }\end{array}$ & NG tube & $\begin{array}{l}1 \text { capsule (Medilac-S, China) } 0.5 \mathrm{~g} \\
\text { three times daily. Each probiotic } \\
\text { capsule contained } \\
\text { active Bacillus subtilis and Enterococcus } \\
\text { faecalis at a concentration of } 4.5 \times \\
10^{9} / 0.25 \mathrm{~g} \text { and } 0.5 \times 10^{9} / 0.25 \mathrm{~g} \\
\text { respectively }\end{array}$ & EN (standard) \\
\hline
\end{tabular}

CFU colony forming units, C.Random concealed randomization, EN enteral nutrition, FOS fructooligosaccharides, NG nasogastric, NJ nasojejunal, NR not reported, OG orogastric, ITT intention to treat, SIRS, systemic inflammatory response syndrome, VAP ventilator-associated pneumonia, BID twice daily. Trevis ${ }^{T M}: 1$ capsule $=$ Lactobacillus acidophilus La5, Bifidobacterium lactis Bb12, Streptococcus thermophilus, Lactobacillus bulgaricus, $4 \times 10^{9} /$ total; Synbiotic 2000 Forte: $10^{11}$ CFU each of Pediococcus pentoseceus 5-33:3, Leuconostoc mesenteroides 32-77:1, L. paracasei ssp paracasei 19, L. plantarum 2362, and 2.5 g each of inulin, oat bran, pectin and resistant starch; Ergyphilus: $10^{10}$ Lactobaccilus rhamnosus GG, Lactobacillus casei, L. acidophilus, Bifidobacterium bifidus; VSL \# $3:>10^{10}$ Bifidobacterium longum, Bifidobacterium breve, $>10^{10 / 9}$ Bifidobacterium infantis, $>10^{11 / 9}$ L. acidophulus, L. plantarum, L. casei, L. bulgaris, and Streptococcus thermophiles; Jinshuangqi: B. longum >10 7 CFU, L. bulgaricus $>10^{6}$ CFU, and S. Thermophilus $>10^{6}$ CFU; Ecologic 641: L. acidophilus, Lactobacillus salivarius, Lactococcus lactis, B. bifidus, and Bifidobacterium lactis; Synbiotic 2000: $10^{10}$ CFU each of P. pentoseceus 5-33:3, Leuconostoc mesenteroides 32-77:1, L. paracasei ssp paracasei 19, L. plantarum 2362, and 2.5 g each of betaglucan, inulin, pectin and resistant starch; Golden Bifid: B. bifidum, L. bulgaricus, and S. thermophilus triple-human probiotic-supplemented oligosaccharides FOS (bifidus factor) 
Table 2 Reported clinical outcomes in RCTs evaluating probiotics in critically ill patients

\begin{tabular}{|c|c|c|c|c|c|c|c|c|c|}
\hline \multirow{2}{*}{\multicolumn{2}{|c|}{ Study }} & \multicolumn{2}{|l|}{ Mortality } & \multicolumn{2}{|l|}{ Infections } & \multicolumn{2}{|l|}{ Length of stay } & \multicolumn{2}{|l|}{ Diarrhea } \\
\hline & & Intervention & Control & Intervention & Control & Intervention & Control & Intervention & Control \\
\hline 1 & Tempe 1983 [30] & $3 / 20(15)$ & $3 / 20(15)$ & NR & NR & NR & NR & $\begin{array}{l}\text { Diarrhea days } \\
34 / 389(9)\end{array}$ & $\begin{array}{l}\text { Diarrhea days } \\
63 / 373(17)\end{array}$ \\
\hline 2 & $\begin{array}{l}\text { Schlotterer } 1987 \\
{[31]}\end{array}$ & $N R$ & $N R$ & $N R$ & $N R$ & $N R$ & $N R$ & $\begin{array}{l}\text { Diarrhea days } \\
3 / 150 \text { (2) }\end{array}$ & $\begin{array}{l}\text { Diarrhea days } \\
19 / 143(13)\end{array}$ \\
\hline 3 & $\begin{array}{l}\text { Heimburger } 1994 \\
\text { [32] }\end{array}$ & $N R$ & $N R$ & $N R$ & $N R$ & NR & $N R$ & Diarrhea 5/16 (31) & Diarrhea 2/18 (11) \\
\hline 4 & Bleichner 1997 [33] & $N R$ & $N R$ & $N R$ & $N R$ & $N R$ & $N R$ & $\begin{array}{l}\text { Diarrhea 18/64 (28) } \\
\text { Days w/diarrhea } \\
\text { 91/648 (14) }\end{array}$ & $\begin{array}{l}\text { Diarrhea 24/64 (38) } \\
\text { Days w/diarrhea } \\
134 / 683 \text { (20) }\end{array}$ \\
\hline 5 & Kecskes 2003 [34] & $\begin{array}{l}\text { Hospital } \\
1 / 22(5)\end{array}$ & $\begin{array}{l}\text { Hospital } \\
2 / 23(9)\end{array}$ & $\begin{array}{l}\text { Septic compl } \\
1 / 22(5)\end{array}$ & $\begin{array}{l}\text { Septic compl } \\
7 / 23(30)\end{array}$ & $\begin{array}{l}\text { Hospital } \\
13.7 \pm 8.7\end{array}$ & $\begin{array}{l}\text { Hospital } \\
21.4 \pm 17.9\end{array}$ & $N R$ & $N R$ \\
\hline 6 & Jain 2004 [36] & $\begin{array}{l}\text { Hospital } \\
\text { 22/45 (49) }\end{array}$ & $\begin{array}{l}\text { Hospital } \\
\text { 20/45 (45) }\end{array}$ & $\begin{array}{l}\text { Septic compl } \\
33 / 45 \text { (73) }\end{array}$ & $\begin{array}{l}\text { Septic compl } \\
26 / 45 \text { (58) }\end{array}$ & $\begin{array}{l}\text { Hospital } \\
24.0 \pm 31.5 \\
\text { ICU } 11.9 \pm 13.1\end{array}$ & $\begin{array}{l}\text { Hospital } \\
18.7 \pm 13.5 \\
\text { ICU } 9.0 \pm 8.9\end{array}$ & $N R$ & NR \\
\hline 7 & Lu 2004 [35] & $\begin{array}{l}\text { Hospital } \\
2 / 20(10)\end{array}$ & $\begin{array}{l}\text { Hospital } \\
1 / 20(5)\end{array}$ & $\begin{array}{l}\text { Infectious compl } \\
8 / 20(40)\end{array}$ & $\begin{array}{l}\text { Infectious compl } \\
11 / 20(55)\end{array}$ & $N R$ & NR & $N R$ & NR \\
\hline 8 & Klarin 2005 [37] & $\begin{array}{l}\text { Hospital } \\
2 / 8(25) \\
\text { ICU } 1 / 8(12)\end{array}$ & $\begin{array}{l}\text { Hospital } \\
2 / 7(29) \\
\text { ICU 2/7 (29) }\end{array}$ & $N R$ & NR & $\begin{array}{l}\text { Hospital } \\
48.3 \pm 30.4 \\
\text { ICU } 14.2 \pm 10.6\end{array}$ & $\begin{array}{l}\text { Hospital } \\
34.3 \pm 15.4 \\
\text { ICU } 16.3 \pm 15.7\end{array}$ & $N R$ & NR \\
\hline 9 & $\begin{array}{l}\text { McNaught } 2005 \\
\text { [38] }\end{array}$ & 18/52 (35) & $18 / 51(35)$ & $\begin{array}{l}\text { Septic morbidity } \\
21 / 52(40)\end{array}$ & $\begin{array}{l}\text { Septic morbidity } \\
22 / 51 \text { (43) }\end{array}$ & ICU 5 (2-9) & ICU 4 (2-7) & $N R$ & NR \\
\hline 10 & $\begin{array}{l}\text { Kotzampassi } 2006 \\
\text { [39] }\end{array}$ & ICU 5/35 (14) & ICU 9/30 (30) & $\begin{array}{l}\text { Infections } \\
22 / 35(63) \\
\text { VAP 19/35 (54) } \\
\text { Septic compl } \\
17 / 35 \text { (49) } \\
\text { Central venous } \\
\text { line infections } \\
13 / 35 \text { (37) } \\
\text { Wound infections } \\
6 / 35 \text { (17) } \\
\text { UTI 6/35 (17) }\end{array}$ & $\begin{array}{l}\text { Infections } 27 / 30(90) \\
\text { VAP } 24 / 30(80) \\
\text { Septic compl } \\
23 / 30(77) \\
\text { Central venous line } \\
\text { infections } 20 / 30 \text { (66) } \\
\text { Wound infections } \\
8 / 30 \text { (26) } \\
\text { UTI 13/30 (43) }\end{array}$ & ICU $27.7 \pm 15.2$ & ICU $41.3 \pm 20.5$ & Diarrhea 5/35 (14) & Diarrhea 10/30 (30) \\
\hline 11 & Alberda 2007 [40] & ICU 1/10 (10) & ICU 1/9 (11) & $N R$ & $N R$ & $N R$ & NR & Diarrhea 1/10 (14) & Diarrhea 2/9 (23) \\
\hline 12 & Li 2007 [41] & NR & $N R$ & Infections 8/14 (58) & Infections 10/11 (91) & $\begin{array}{l}\text { Hospital } \\
42 \pm 5.0\end{array}$ & $\begin{array}{l}\text { Hospital } \\
49 \pm 6.8\end{array}$ & $N R$ & NR \\
\hline
\end{tabular}


Table 2 Reported clinical outcomes in RCTs evaluating probiotics in critically ill patients (Continued)

\begin{tabular}{|c|c|c|c|c|c|c|c|c|c|}
\hline 13 & Olah 2007 [42] & $\begin{array}{l}\text { Hospital } \\
2 / 33(6)\end{array}$ & $\begin{array}{l}\text { Hospital } \\
6 / 29(21)\end{array}$ & $\begin{array}{l}\text { Infections 9/33 (27) } \\
\text { Septic compl } \\
7 / 33(12) \\
\text { Pancreatic abscess } \\
2 / 33(6) \\
\text { Infected } \\
\text { pancreatic } \\
\text { necrosis 2/33 (6) } \\
\text { UTI 3/33 (9) }\end{array}$ & $\begin{array}{l}\text { Infections 15/29 (52) } \\
\text { Septic compl } \\
17 / 29(28) \\
\text { Pancreatic abscess } \\
2 / 29(7) \\
\text { Infected pancreatic } \\
\text { necrosis 6/29 (21) } \\
\text { UTI 3/33 (9) }\end{array}$ & $\begin{array}{l}\text { Hospital } \\
14.9 \pm 3.3\end{array}$ & $\begin{array}{l}\text { Hospital } \\
19.7 \pm 4.5\end{array}$ & NR & NR \\
\hline 14 & Forestier 2008 [44] & NR & NR & VAP 19/102 (19) & VAP 21/106 (20) & ICU $22.5 \pm 20.6$ & ICU $19.7 \pm 16.7$ & NR & NR \\
\hline 15 & Besselink 2008 [43] & 24/152 (16) & 9/144 (6) & $\begin{array}{l}\text { Infections } \\
46 / 152(30) \\
\text { VAP 24/152 (16) } \\
\text { Bacteremia } \\
33 / 152(22) \\
\text { Infected necrosis } \\
21 / 152(14) \\
\text { Urosepsis 1/52 (2) }\end{array}$ & $\begin{array}{l}\text { Infections } 41 / 144 \text { (28) } \\
\text { VAP 16/144 (11) } \\
\text { Bacteremia } \\
22 / 144(15) \\
\text { Infected necrosis } \\
14 / 144(10) \\
\text { Urosepsis 2/144 (1) }\end{array}$ & $\begin{array}{l}\text { Hospital } \\
28.9 \pm 41.5 \\
\text { ICU } 6.6 \pm 17\end{array}$ & $\begin{array}{l}\text { Hospital } \\
23.5 \pm 25.9 \\
\text { ICU } 3.0 \pm 9.3\end{array}$ & $\begin{array}{l}\text { Diarrhea } \\
\text { 25/152 (16) }\end{array}$ & $\begin{array}{l}\text { Diarrhea } \\
\text { 28/144 (19) }\end{array}$ \\
\hline 16 & Klarin 2008 [45] & $\begin{array}{l}\text { Hospital } \\
3 / 22(5) \\
\text { ICU 2/22 (9) }\end{array}$ & $\begin{array}{l}\text { Hospital } \\
2 / 22(0) \\
\text { ICU 2/22 (9) }\end{array}$ & $\begin{array}{l}\text { C. difficile + fecal } \\
\text { samples } 0 / 71\end{array}$ & $\begin{array}{l}\text { C. difficile + fecal } \\
\text { samples } 4 / 80\end{array}$ & $\begin{array}{l}\text { Hospital } \\
25.8 \pm 19.4 \\
\text { ICU } 8.0 \pm 5.4\end{array}$ & $\begin{array}{l}\text { Hospital } \\
50.3 \pm 75.2 \\
\text { ICU } 11.6 \pm 14\end{array}$ & NR & NR \\
\hline 17 & Knight 2009 [46] & $\begin{array}{l}\text { Hospital } \\
35 / 130(27) \\
\text { ICU 28/130 (22) }\end{array}$ & $\begin{array}{l}\text { Hospital } \\
42 / 129 \text { (33) } \\
\text { ICU 34/129 (26) }\end{array}$ & VAP 12/130 (9) & VAP $17 / 129$ (13) & ICU 6 (3-11) & ICU 7 (3-14) & $\begin{array}{l}\text { Diarrhea } \\
7 / 130(5)\end{array}$ & $\begin{array}{l}\text { Diarrhea } \\
\text { 9/129 (7) }\end{array}$ \\
\hline 18 & Barraud 2010 [47] & $\begin{array}{l}\text { ICU } 21 / 87 \text { (24) } \\
28 \text { days } \\
22 / 87(25) \\
90 \text { days } \\
27 / 87(31)\end{array}$ & $\begin{array}{l}\text { ICU } 21 / 80 \text { (26) } \\
28 \text { days } \\
19 / 80(24) \\
90 \text { days } \\
24 / 80(30)\end{array}$ & $\begin{array}{l}\text { All infections } \\
30 / 87(34) \\
\text { Infection > 96 h } \\
26 / 87(30) \\
\text { VAP 23/87 (26) } \\
\text { Catheter-related } \\
\text { BSI 3/87 (4) } \\
\text { UTI 4/87 (5) }\end{array}$ & $\begin{array}{l}\text { All infections } \\
30 / 80(38) \\
\text { Infection > } 96 \mathrm{~h} \\
29 / 80(36) \\
\text { VAP } 15 / 80(19) \\
\text { Catheter-related BSI } \\
11 / 80(14) \\
\text { UTI } 4 / 89(5)\end{array}$ & $\begin{array}{l}\text { Hospital } \\
26.6 \pm 22.3 \\
\text { ICU } 18.7 \pm 12.4\end{array}$ & $\begin{array}{l}\text { Hospital } \\
28.9 \pm 26.4 \\
\text { ICU } 20.2 \pm 20.8\end{array}$ & $\begin{array}{l}\text { Diarrhea } \\
48 / 87(55)\end{array}$ & $\begin{array}{l}\text { Diarrhea } \\
\text { 42/80 (53) }\end{array}$ \\
\hline 19 & Morrow 2010 [10] & 12/68 (18) & $15 / 70(21)$ & VAP 13/73 (18) & VAP 28/73 (38) & $\begin{array}{l}\text { Hospital } \\
21.4 \pm 14.9 \\
\text { ICU } 14.8 \pm 11.8\end{array}$ & $\begin{array}{l}\text { Hospital } \\
21.7 \pm 17.4 \\
\text { ICU } 14.6 \pm 11.6\end{array}$ & $\begin{array}{l}\text { Non C. difficile } \\
\text { diarrhea } 42 / 68 \text { (62) } \\
\text { C. difficile diarrhea } \\
4 / 68 \text { (6) }\end{array}$ & $\begin{array}{l}\text { Non C. difficile } \\
\text { diarrhea } 44 / 70 \text { (63) } \\
\text { C. difficile diarrhea } \\
13 / 70 \text { (19) }\end{array}$ \\
\hline 20 & $\begin{array}{l}\text { Frohmader } 2010 \\
\text { [48] }\end{array}$ & $5 / 20(25)$ & $3 / 25(12)$ & NR & NR & ICU $7.3 \pm 5.7$ & ICU $8.1 \pm 4$ & $\begin{array}{l}\text { Diarrhea episodes/ } \\
\text { pt/day } 0.53 \pm 0.54\end{array}$ & $\begin{array}{l}\text { Diarrhea episodes/ } \\
\text { pt/day } 1.05 \pm 1.08\end{array}$ \\
\hline 21 & Ferrie 2011 [49] & $\begin{array}{l}\text { Hospital } \\
2 / 18(11) \\
6 \text { months } \\
7 / 18(39)\end{array}$ & $\begin{array}{l}\text { Hospital } \\
2 / 18(11) \\
6 \text { months } \\
5 / 18(28)\end{array}$ & $\begin{array}{l}\text { Infections } \\
14 / 18(78)\end{array}$ & $\begin{array}{l}\text { Infections } \\
16 / 18 \text { (89) }\end{array}$ & $\begin{array}{l}\text { Hospital } \\
54.50 \pm 31.26 \\
\text { ICU } 32.04 \pm 24.46\end{array}$ & $\begin{array}{l}\text { Hospital } \\
59.04 \pm 33.92 \\
\text { ICU } 29.75 \pm 18.81\end{array}$ & $\begin{array}{l}\text { Duration of diarrhea } \\
3.83 \pm 2.39 \\
\text { Loose stools/day } \\
1.58 \pm 0.88\end{array}$ & $\begin{array}{l}\text { Duration of diarrhea } \\
2.56 \pm 1.85 \\
\text { Loose stools/day } \\
1.10 \pm 0.79\end{array}$ \\
\hline 22 & Sharma 2011 [50] & $\begin{array}{l}\text { Hospital } \\
2 / 24(8)\end{array}$ & $\begin{array}{l}\text { Hospital } \\
2 / 26(8)\end{array}$ & NR & $N R$ & $\begin{array}{l}\text { Hospital } \\
13.23 \pm 18.19 \\
\text { ICU } 4.94 \pm 9.54\end{array}$ & $\begin{array}{l}\text { Hospital } \\
9.69 \pm 9.69 \\
\text { ICU } 4.0 \pm 5.86\end{array}$ & NR & NR \\
\hline
\end{tabular}


Table 2 Reported clinical outcomes in RCTs evaluating probiotics in critically ill patients (Continued)

\begin{tabular}{|c|c|c|c|c|c|c|c|c|c|}
\hline 23 & Tan 2011 [51] & $\begin{array}{l}28 \text { days } \\
3 / 26(12)\end{array}$ & $\begin{array}{l}28 \text { days } \\
5 / 26 \text { (19) }\end{array}$ & $\begin{array}{l}\text { Infections } \\
9 / 26(35) \\
\operatorname{VAP} 7 / 26(27)\end{array}$ & $\begin{array}{l}\text { Infections 15/26 (58) } \\
\text { VAP } 13 / 26(50)\end{array}$ & ICU $6.8 \pm 3.8$ & ICU $10.7 \pm 7.3$ & NR & NR \\
\hline 24 & Cui 2013 [20] & $\begin{array}{l}\text { Hospital } \\
1 / 23(4)\end{array}$ & $\begin{array}{l}\text { Hospital } \\
1 / 25(4)\end{array}$ & N/A & N/A & $\begin{array}{l}\text { Hospital } \\
10.4 \pm 3.9 \text { (23) }\end{array}$ & $\begin{array}{l}\text { Hospital } \\
13.4 \pm 5.2(25)\end{array}$ & NR & NR \\
\hline 25 & Tan 2013 [21] & $\begin{array}{l}28 \text { days } \\
23 / 26(12)\end{array}$ & $\begin{array}{l}28 \text { days } \\
5 / 26(19)\end{array}$ & NR & NR & ICU $6.8 \pm 3.8$ (26) & ICU $10.7 \pm 7.3(26)$ & NR & $N R$ \\
\hline 26 & Wang 2013 [22] & $\begin{array}{l}\text { Unspecified } \\
1 / 62(8.1)\end{array}$ & $\begin{array}{l}\text { Unspecified } \\
3 / 61(9.8)\end{array}$ & $\begin{array}{l}\text { Pancreatic sepsis } \\
8 / 62(13) \\
\text { MODS } 7 / 62(11.3)\end{array}$ & $\begin{array}{l}\text { Pancreatic sepsis } \\
13 / 61(21) \\
\text { MODS 15/61 (25) }\end{array}$ & NR & NR & NR & NR \\
\hline 27 & $\begin{array}{l}\text { Lopez de Toro } \\
2014 \text { [23] }\end{array}$ & $\begin{array}{l}\text { Hospital } \\
\text { 19/46 (41) } \\
\text { ICU 15/46 (33) }\end{array}$ & $\begin{array}{l}\text { Hospital } \\
\text { 18/43 (42) } \\
\text { ICU } 14 / 43 \text { (33) }\end{array}$ & $\begin{array}{l}\text { Hospital acquired } \\
\text { infections } \\
9 / 46(20)\end{array}$ & $\begin{array}{l}\text { Hospital acquired } \\
\text { infections } \\
13 / 43(30)\end{array}$ & $\begin{array}{l}\text { Hospital } \\
18.5(10-36) \\
\text { ICU } 9(3-19)\end{array}$ & $\begin{array}{l}\text { Hospital } \\
24.5(10-38) \\
\text { ICU } 8(2.5-16.5)\end{array}$ & NR & NR \\
\hline 28 & Sanaie 2014 [24] & $\begin{array}{l}28 \text { days } \\
2 / 20(10)\end{array}$ & $\begin{array}{l}28 \text { days } \\
5 / 20(25)\end{array}$ & $\begin{array}{l}\text { Bacteremia } \\
2 / 20(10)\end{array}$ & $\begin{array}{l}\text { Bacteremia } \\
5 / 20(25)\end{array}$ & ICU $13.85 \pm 6.96$ & ICU $14.16 \pm 5.97$ & NR & NR \\
\hline 29 & $\begin{array}{l}\text { Rongrungruang } \\
2015 \text { [25] }\end{array}$ & $\begin{array}{l}28 \text { days } \\
18 / 75 \text { (24) } \\
90 \text { day } \\
25 / 75(33)\end{array}$ & $\begin{array}{l}28 \text { days } \\
17 / 75 \text { (22.7) } \\
90 \text { day } \\
26 / 75 \text { (34.7) }\end{array}$ & VAP $18 / 75$ (24) & VAP $22 / 75$ (29.3) & $\begin{array}{l}\text { Hospital } \\
20(2-106) \\
\text { ICU } 30.5(4-98)\end{array}$ & $\begin{array}{l}\text { Hospital } \\
19(3-171) \\
\text { ICU } 19(5-30)\end{array}$ & $\begin{array}{l}\text { Diarrhea } \\
\text { 19/75 (25.3) }\end{array}$ & $\begin{array}{l}\text { Diarrhea } \\
14 / 75(18.7)\end{array}$ \\
\hline 30 & Zeng 2016 [26] & $\begin{array}{l}\text { Hospital } \\
26 / 118(22) \\
\text { ICU } 15 / 118(12.7)\end{array}$ & $\begin{array}{l}\text { Hospital } \\
25 / 117(21.4) \\
\text { ICU 9/117 (7.7) }\end{array}$ & VAP $43 / 118$ (36.4) & VAP 59/117 (50.4) & $\begin{array}{l}\text { Hospital } \\
13.5 \pm 12.4 \\
\text { ICU } 18(14-32)\end{array}$ & $\begin{array}{l}\text { Hospital } \\
10.6 \pm 10.2 \\
\text { ICU } 22(11-56)\end{array}$ & NR & NR \\
\hline
\end{tabular}

BSI blood stream infection, ICU intensive care unit, NR not reported, $R C T$ randomized control trial, UTI urinary tract infection, VAP ventilator associated pneumonia, N/A non-attributable, compl complications, MODS Multiple Organ Dysfunction Syndrome, Days w/ diarrhea - days with diarrhea 
reduction in infections (RR $0.80,95 \%$ CI 0.68, 0.95, $P=0.009$; heterogeneity $I^{2}=36 \%, P=0.09$; Fig. 1 ).

\section{Secondary outcomes}

\section{Ventilator associated pneumonia}

Aggregating the data from 9 trials that reported VAP, there was a significant reduction in the incidence of VAP (RR 0.74, $95 \%$ CI 0.61, 0.90, $P=0.002 ; \mathrm{I}^{2}=19 \%$, $P=0.27$; Fig. 2).

\section{Overall effect on mortality}

A total of 17 studies reported on hospital mortality. When statistically aggregated, probiotic therapy did not significantly affect mortality (RR 0.98, $95 \%$ CI 0.82, 1.18, $P=0.85 ; I^{2}=0 \%$, Fig. 3). Moreover, probiotics did not show any effect on ICU mortality (RR 0.90, $95 \%$ CI $\left.0.70,1.17, P=0.44 ; I^{2}=0 \%\right)$.

\section{Overall effect on ICU length of stay}

Aggregating the data from the 14 RCTs reporting on ICU LOS, there were no significant differences between the groups (weighted mean difference (WMD) -3.26, $95 \%$ CI -7.82, 1.31, $\left.P=0.16 ; I^{2}=93 \%, P<0.00001\right)$.

\section{Overall effect on hospital length of stay}

Aggregating the data from the nine RCTs that reported hospital LOS, there were no significant differences between the groups (WMD -0.58, $95 \%$ CI -3.66, 2.50, $\left.P=0.71 ; I^{2}=74 \%, P<0.00001\right)$.

\section{Diarrhea}

Aggregating the data from nine trials that reported on diarrhea, probiotics had no effect (RR 0.97; $95 \%$ CI 0.82, $1.15 ; P=0.74 ; I^{2} 5 \%, P=0.39$; Fig. 4$)$.

\section{Antibiotic days}

When we aggregated the data of four trials reporting on antibiotic days, we found that probiotics were significantly associated with a reduction in the duration of antibiotic therapy (WMD -1.12, $95 \%$ CI -1.72, -0.51, $P=0.0003$; $I^{2}=32 \%, P=0.22$; Fig. 5).

\section{Subgroup analysis \\ Probiotics daily dose}

There were similar rates of infectious complications in RCTs using high-dose probiotic therapy ( $\mathrm{n}=8$ trials) $\left(0.87 ; 95 \%\right.$ CI $\left.0.72-1.06 ; P=0.18 ; I^{2}=43 \%\right)$ and in those using lower daily doses $(\mathrm{n}=2$ trials) $\mathrm{RR} 0.40 ; 95 \% \mathrm{CI}$ $0.11-1.50 ; P=0.18 ; I^{2}=48 \%$. The difference between subgroups was not significant, $P=0.25$ ).

\section{L. plantarum vs. non L. plantarum}

Subgroup analyses showed that four trials administering L. plantarum, either alone or in combination with other probiotics, were associated with a significant reduction in overall infections $(\mathrm{RR}=0.70,95 \% \mathrm{CI} 0.50,0.97 ; P=$ $\left.0.03 ; I^{2}=36 \%\right)$. However, in the 10 trials that did not include $L$. plantarum, there was no significant effect on overall infectious complications ( $\mathrm{RR}=0.88,95 \% \mathrm{CI}$ $\left.0.74,1.04 ; P=0.15 ; I^{2}=21 \%\right)$. Test for subgroup differences between groups was not significant $(P=0.21)$.

\section{L. rhamnosus $G G$ vs. other probiotics}

In two trials using LGG there was no significant effect on reduction in infectious complications (RR 0.86; $95 \%$ CI $0.67-1.10 ; P=0.22 ; I^{2}=0 \%$ ). However, in 12 trials that supplemented other probiotics there was a significant reduction in overall infections (RR 0.77; $95 \% \mathrm{CI}$ $\left.0.62-0.95 ; P=I^{2}=45 \%\right) ; P=0.52$ for the difference between groups.

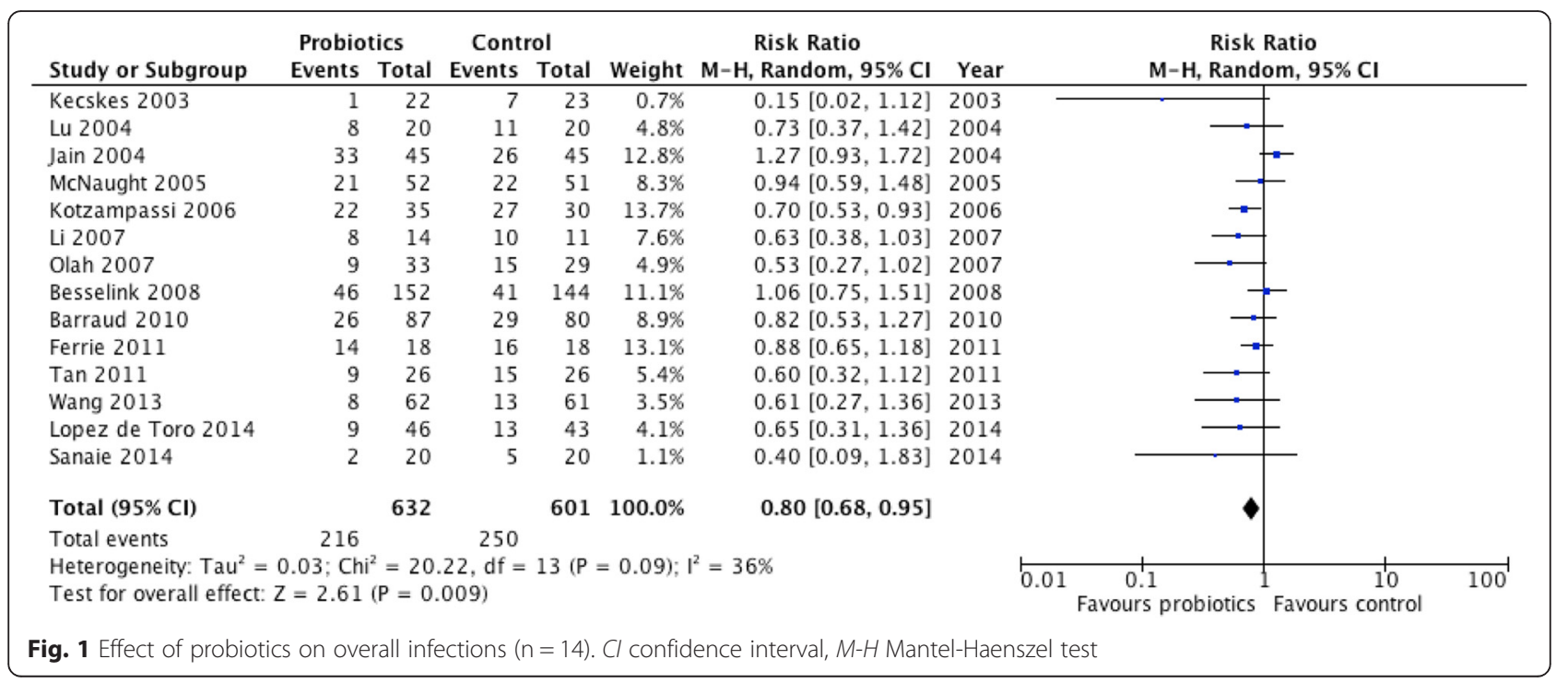




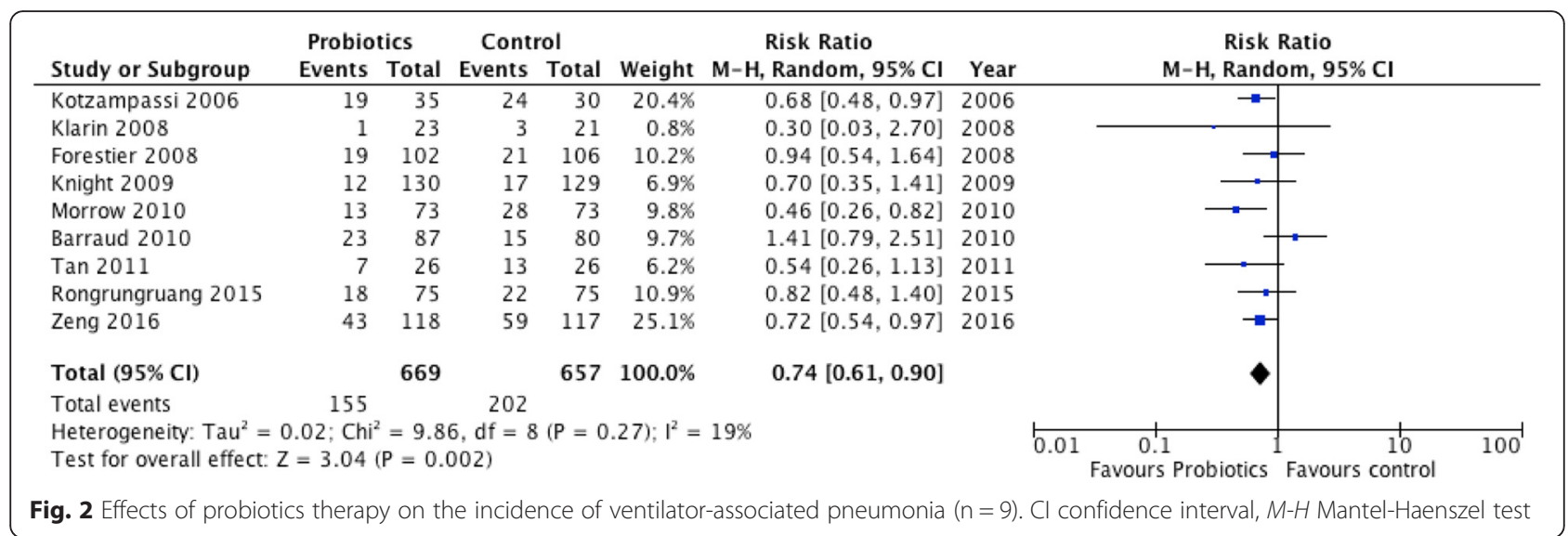

\section{Synbiotics vs. other strategies}

In subgroup analyses of the four trials that administered synbiotics there was no effect on infections $(R R=0.80$, $95 \%$ CI 0.49, 1.30, $P=0.36 ; I^{2}=66 \%, P=0.03$ ) (Fig. 6). However, in 10 studies that administered probiotics alone there was a significant reduction in the incidence of infections (RR 0.79, $95 \%$ CI 0.68, 0.92, $P=0.002 ; I^{2}=9 \%, P=$ 0.36). The $P$ value for subgroup differences was not significant $(\mathrm{P}=0.98)$. (Fig. 6).

\section{Higher vs. lower mortality}

The median hospital mortality rate of all the trials (or ICU mortality when hospital mortality was not reported) in the control group was $19 \%$. After aggregating nine studies with a higher mortality rate, probiotics significantly reduced the incidence of infections (RR 0.74; $95 \%$ CI $0.57,0.96 ; P=0.02 ; I^{2}=58 \%, P=0.01$ ) (Fig. 7). However, probiotics did not have an effect on infections in the five studies with lower mortality (RR 0.85; $95 \% \mathrm{CI}$ $0.66,1.11 ; P=0.24 ; I^{2}=23 \%, P=0.27$ ). The test for subgroup differences was not significant $(P=0.43)$ (Fig. 7$)$.

\section{Higher vs. lower methodological score}

The median methodological score was 9.5. In six trials with a higher score $(\geq 9.5)$ there was no effect on infections (RR 0.93; $95 \%$ CI 0.76, 1.15; $P=0.51 ; I^{2}=35 \%, P=0.17$ ), whereas in eight trials with a lower methodological score $(<9.5)$ there was a significant reduction in infectious complications (RR 0.69, $95 \%$ CI 0.57, 0.83, P<0.0001; $I^{2}=$ $0 \%$ ) (Fig. 8); the overall test for subgroup differences was significant for these subgroups $(P=0.03)$.

\section{Publication bias}

There was indication that potential publication bias influenced the observed aggregated results. In fact, funnel plots were created for each study outcome and the tests

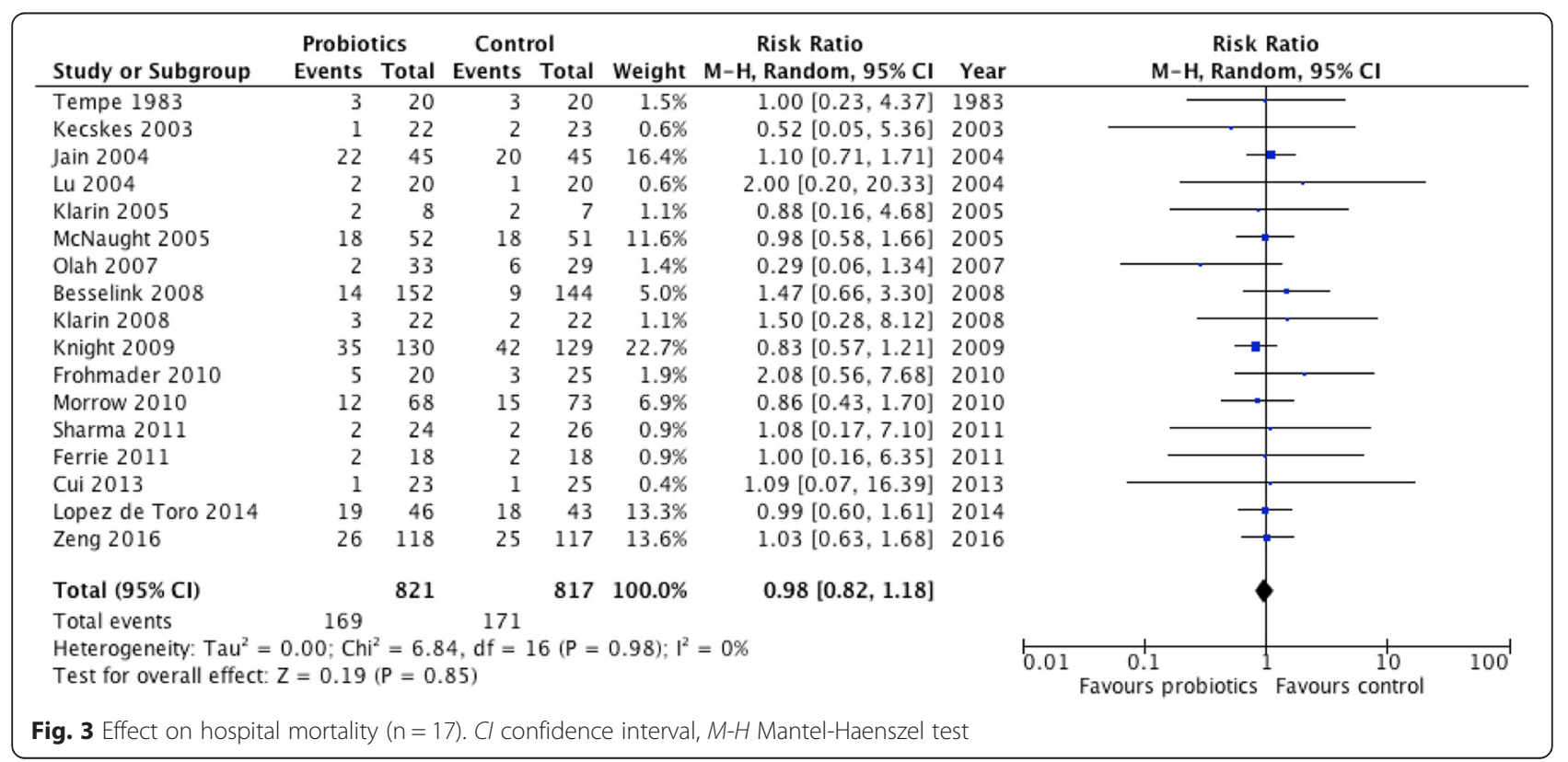




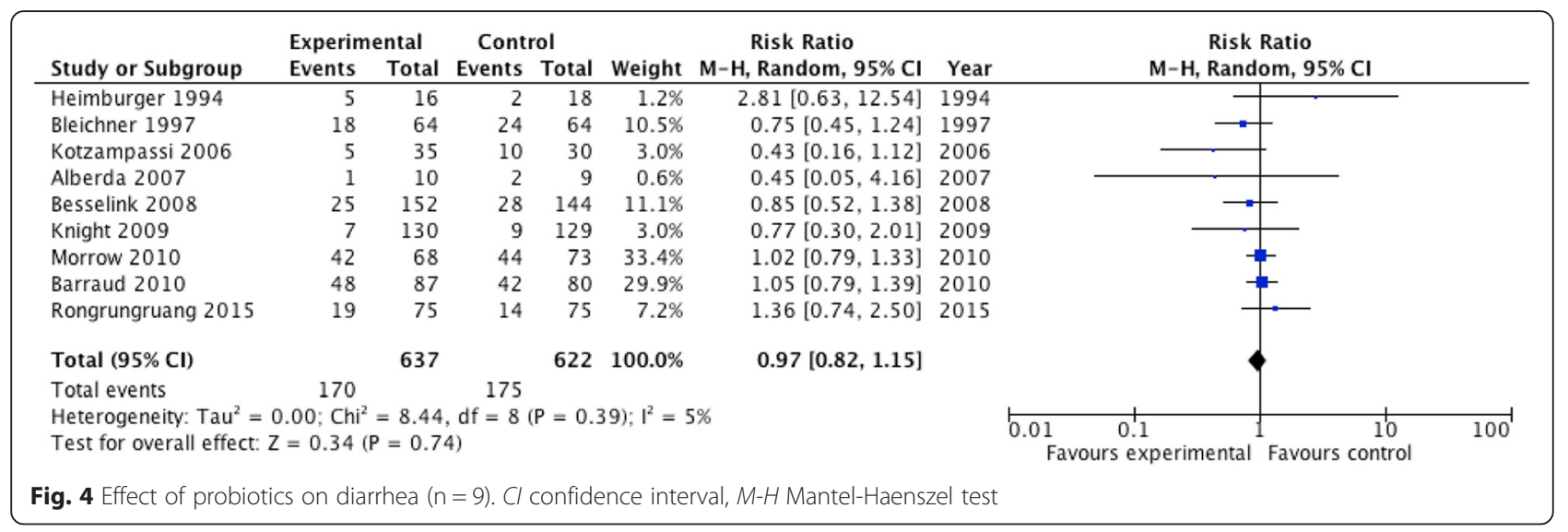

of asymmetry were significant for overall new infections (odds ratio (OR) -2.30, $95 \% \mathrm{CI}-3.56,-1.05, P=0.001$; Fig. 9) and hospital LOS (OR -3.32, $95 \%$ CI -6.12, -0.52 , $P=0.024)$. However, the test for asymmetry was not significant for any other outcome (VAP, $P=0.76$; mortality, $P=0.80$; ICU LOS, $P=0.47$; diarrhea, $P=0.18$ ).

\section{Discussion}

To date, our systematic review and meta-analysis is the largest and most updated evaluation of the overall effects of probiotics in the critically ill. It is also the first to include an analysis of symbiotic (probiotic/fiber combinations). Based on the analysis of 30 trials enrolling 2972 patients we demonstrated that probiotics are associated with a significant reduction in ICU-acquired infections, including VAP, which is the most common infectious complication in the critically ill. This significant effect on VAP is a new finding from our previous systematic reviews. Further, the beneficial effect of probiotics on reduction of infections is stronger with the publication of the new trials and the data no longer show a statistically significant effect of heterogeneity on this endpoint. Despite the probiotic effect of reducing infectious complications, this therapy did not influence ICU or hospital mortality, although none of the trials were powered to detect an effect on mortality. Overall, there was a tendency towards a reduction in ICU LOS and probiotic therapy did not influence other clinical endpoints such as hospital LOS, and diarrhea. Statistical and clinical heterogeneity was observed for some endpoints, although this was significant for the key endpoints of infectious complications and VAP. In addition, publication bias for overall infections and hospital LOS means that larger, well-powered, and more definitive clinical trials are urgently needed aimed to avoid these biases. Moreover, subgroup analysis showed that those trials with lower methodological quality exhibit the best treatment effect, which is another issue indicating that larger, welldesigned studies are needed. Again, with the exception of four trials, most of the included studies $(n=14)$ that reported mortality had small sample sizes, and hence were underpowered and inadequate to detect any clinically important treatment effects of probiotic therapy on mortality. Moreover, the inferences we can make from our current findings are further weakened, as randomization was concealed in $30 \%$ of trials, whereas double-blinding was performed in $67 \%$ of trials.

Over recent years, several systematic reviews and meta-analyses have been conducted, although our metaanalysis is the largest and most current to date, as it contains the seven new suitable trials published since the most recent comprehensive meta-analysis publication on this topic, which focused on overall infections and other outcomes (not primarily VAP) in 2012 [14]. Further, these previous systematic reviews did not include analysis of synbiotic therapy. Overall, we have examined several

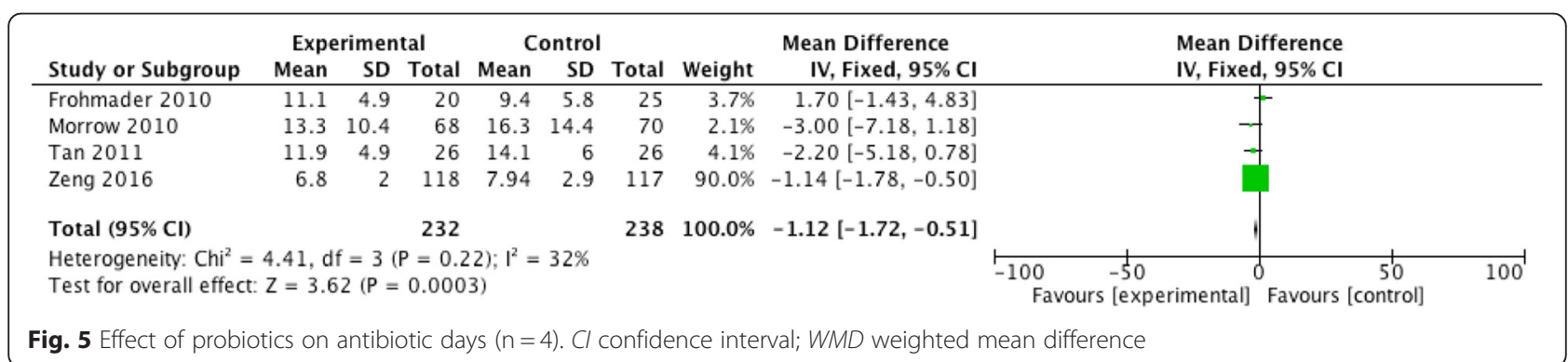




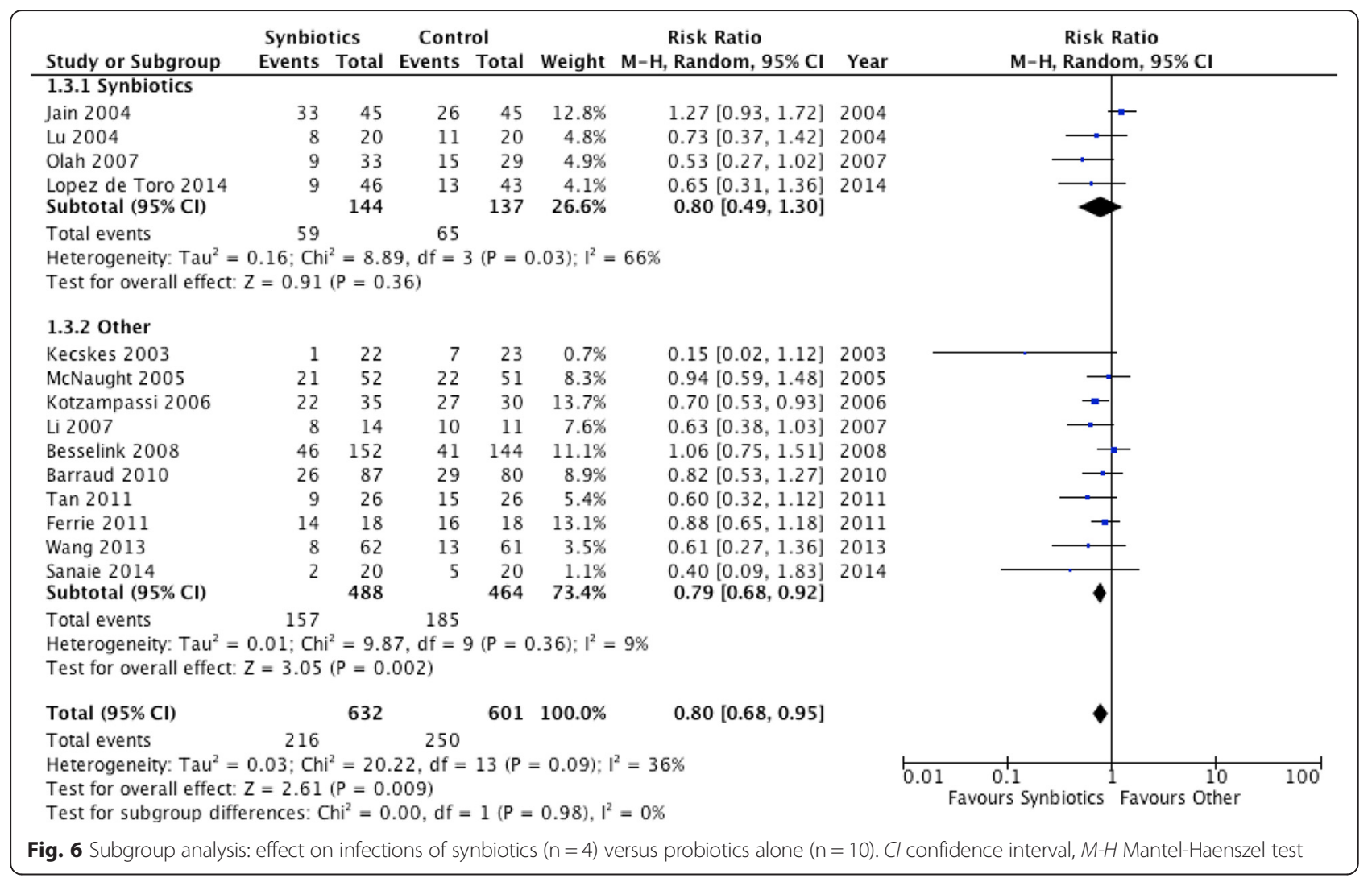

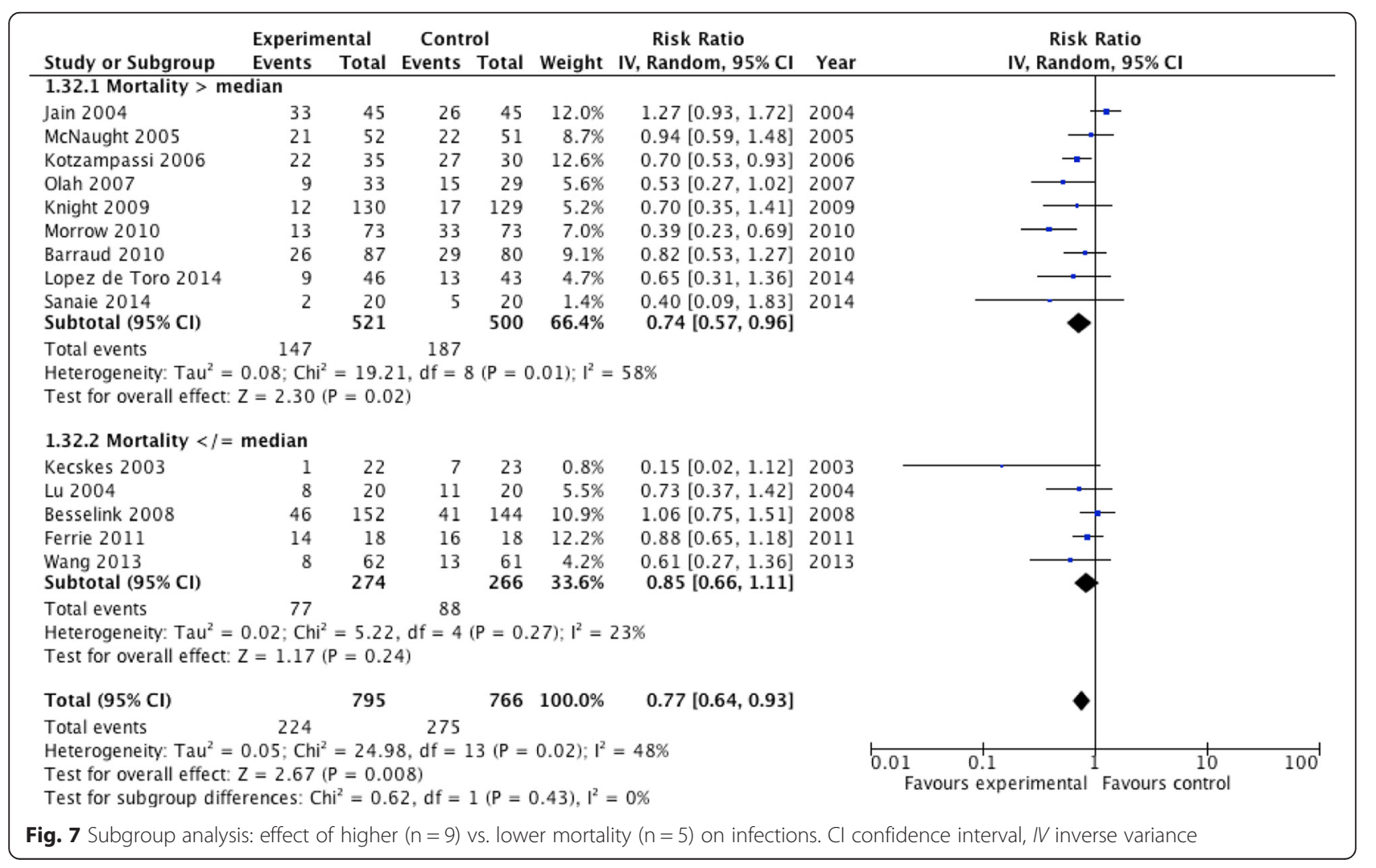




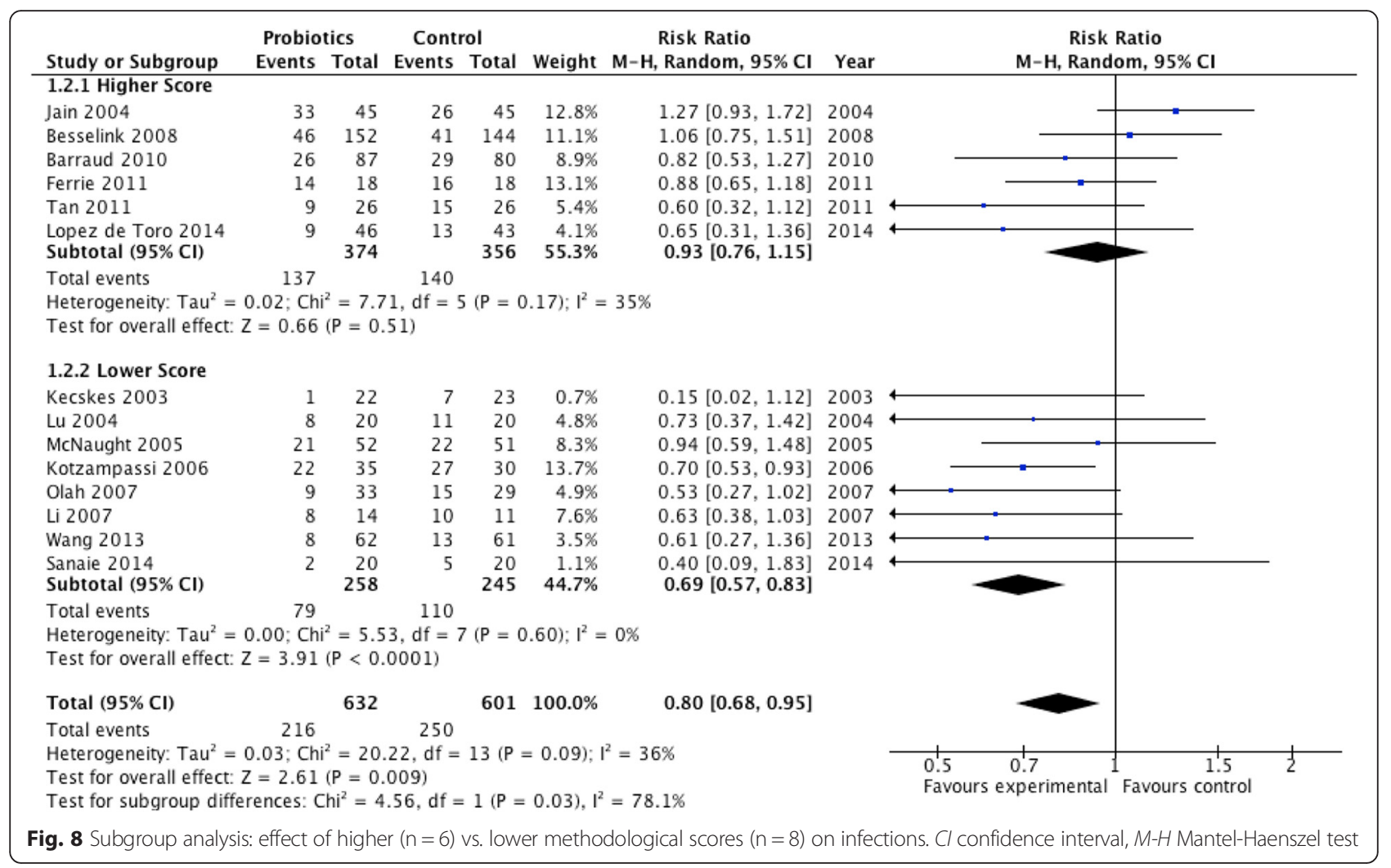

relevant clinical outcomes in a heterogenous ICU patient population, and therefore our results could be applied to a broad group of critically ill patients with sepsis, trauma, severe pancreatitis, or who have undergone surgery. Specific to pancreatitis, concerns have been raised about the safety of probiotic therapy following the 2008 trial, Probiotic prophylaxis in patients with predicted severe acute pancreatitis (PROPATRIA) [43], which showed that
Ecologic $641^{\odot}$ given with fiber post-pyloric was associated with higher mortality and bowel ischemia. This postpyloric method of administration was associated with an increase in small bowel necrosis, which was subsequently associated with death in a number of patients receiving the prebiotic fiber/probiotic mixture. It is possible that the post-pyloric administration of this fiber/multiple probiotic strain mixture in patients with pancreatitis may carry

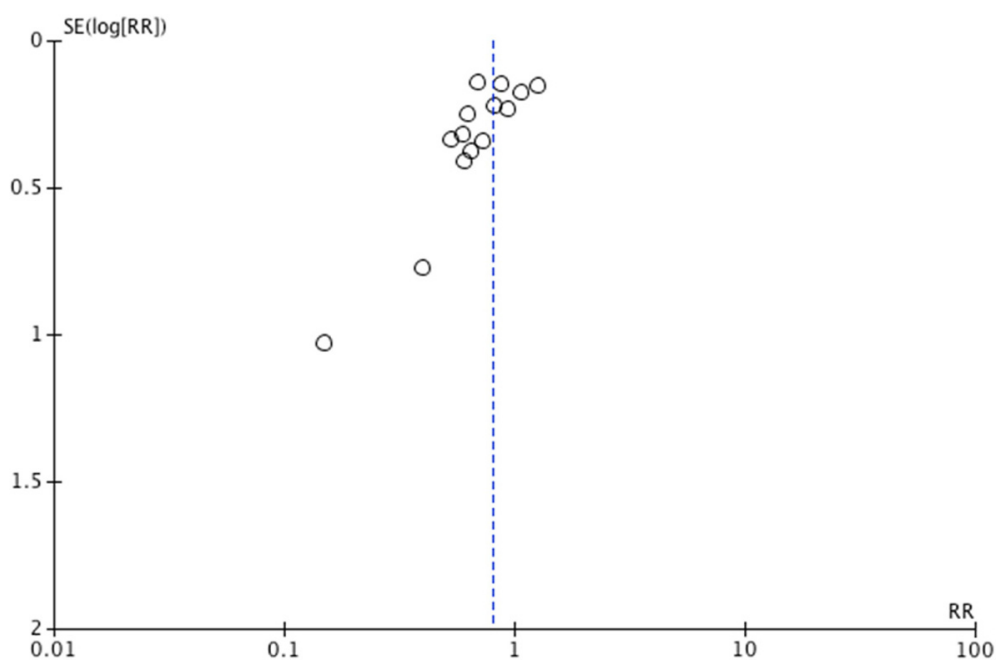

Fig. 9 Funnel plot of primary outcome $(n=14)$. Overall new infections (test for asymmetry, OR $-2.30,95 \% \mathrm{Cl}-3.56,-1.05, P=0.001$ ). OR odds ratio 
significant risk and should likely be avoided [52]. Unfortunately, there were significant ethical and statistical concerns raised about the conduct of the trial [53], limiting the utility of the data. Further, more recently, a systematic review and meta-analysis by Gou et al. [54] found that probiotics had neither beneficial nor adverse effects in patients with pancreatitis.

Despite the limitations of the PROPRIATA trial, it has contributed to concerns around the safety of probiotic administration in critical illness and limited the design of larger-scale clinical trials and/or more routine clinical administration of live probiotics. To address this, the American Health Care Research and Quality (AHRQ) agency reviewed and reported on the safety of probiotic therapy in over 600 published clinical trials and case reports [55]. It should be reassuring to future investigators that the overall conclusion of the extensive AHRQ report indicates that probiotic therapy in both adults and pediatric populations was not been found to be associated with any increased risk of infectious or other adverse events in either healthy or ill patients. Importantly, their report revealed a trend towards less adverse events in probiotic-treated critically ill patients, although isolated adverse effects of probiotic administration have been reported [56]. In any case, careful and appropriate safety monitoring in all future probiotic clinical trials should be conducted.

Recent data indicate that infection during critical illness continues to be a major challenge worldwide. A multi-national ICU study of 14,414 patients in 1265 ICUs from 75 countries, revealed that $51 \%$ of ICU patients were considered infected on the day of survey and $71 \%$ were receiving antibiotics [57]. Of the infections in this study, $64 \%$ were of respiratory origin and the ICU mortality rate in infected patients was more than twice that of non-infected patients ( $25 \%$ vs. $11 \%, P<0.001)$, as was the hospital mortality rate (33\% infected vs. $15 \%$ non-infected, $P<0.001$ ) [57].

Currently, VAP is the second most common nosocomial infection in the USA and the most prevalent ICUacquired infection. Notwithstanding, its incidence is highly variable depending on diagnostic criteria used to identify this infectious complication. In fact, in 2015 Ego et al. [58] reported that the incidence of VAP ranged from $4 \%$ to $42 \%$ when using the six published sets of criteria and from $0 \%$ to $44 \%$ when using the 89 combinations of criteria for hypoxemia, inflammatory response, bronchitis, chest radiography, and microbiologic findings. In our systematic review we found that the incidence of VAP ranged from $9 \%$ [46] to $80 \%$ [39]. Additionally, the apparent effect of probiotics on VAP is largely driven by the studies of Kotzampassi et al. [39] study and the Zeng et al. [26]; both trials explain $45.5 \%$ of the signal and thus, provide an unstable estimate. Moreover, current knowledge shows that VAP is associated with high cost and poor clinical outcomes [59]. In 2002, Rello et al. [60] demonstrated that VAP leads to an additional US $\$ 40,000$ in hospital charges per patient, and recently it has been suggested that the use of prophylactic probiotics may be cost-effective for prevention of VAP from a hospital perspective [61].

Probiotic therapy may prevent VAP and other infections by restoring non-pathogenic flora, which competes with nosocomial pathogens inhibiting their overgrowth, modulating local and systemic immune response, and improving gut barrier function. However, in spite of these protective effects the role of probiotics as a nonpharmacological strategy in preventing VAP has previously been inconclusive. In 2010, Siempos et al. [12] aggregated five probiotic trials demonstrating a reduction in the incidence of VAP, whereas in 2012 Petrof et al. [14] and subsequently Barraud et al. [13] and Wang et al. [15] did not demonstrate any significant effect of probiotic therapy on VAP. More recently, a Cochrane review of probiotic therapy specifically for VAP [17], found with low quality of evidence that probiotic therapy is associated with a reduction in the incidence of VAP. Our current systematic review demonstrates a significant treatment effect of probiotics in reducing VAP and did not demonstrate statistical heterogeneity, strengthening the signal that this may be an effective therapy for VAP. Recently, a Canadian survey [27] on the use of probiotics as a prophylactic strategy for VAP showed that most Canadian ICU pharmacists have used probiotics at least once, although they do not routinely recommend probiotics for the prevention of VAP.

Currently, a large number of clinical trials have demonstrated that probiotics may reduce the incidence of antibiotic-associated diarrhea and Clostridium difficile infections, and systematic reviews have confirmed a significant signal of benefit on reduction of diarrhea and $C$. difficile-related colitis in all patients (not confined to ICU patients) [62, 63]. Our results, when focused on ICU patients do not currently demonstrate a treatment benefit of probiotics in preventing and treating diarrhea in the critically ill, including antibioticassociated diarrhea.

An interesting finding of our meta-analysis was a reduction in antibiotic use in those patients who received probiotics. Nonetheless, only four trials $[10,21,26,48]$ comprising $13 \%$ of included studies reported duration of antibiotic therapy as an outcome. In addition, the study of Zeng et al. contributed to $90 \%$ of the signal, which is a very unstable estimate that weakens our finding. Therefore, probiotics may shorten the duration of antibiotic therapy, although the limited clinical trial data available for this endpoint limits the strength of these findings and further investigation of this effect is needed. 
We currently have a greater understanding about the potential benefits of probiotics therapy in critical illness, although much more data are needed. Subgroup analysis found that certain strains such as L. plantarum alone or in combination was associated with a significant reduction in overall infections, although the test for subgroup differences was not significant $(P=0.21)$. Certain specific biological properties have been described for $L$. plantarum, including an ability to prevent adhesion of pathogens to the intestinal epithelium secondary to the production of adhesins, enolase, and phosphoglycerate kinase on the bacterial surface $[64,65]$. These mechanisms may be crucial to reduction of bacterial translocation and modulation of local inflammatory response, and therefore the effect of this strain on systemic infectious complications. Interestingly, probiotics alone had a greater effect than synbiotics on infections, although the difference between these subgroups was not significant $(P=0.98)$ and more data on the specific effects of different prebiotic fibers are needed. Finally, future trials also need to focus on evaluating the changes in the microbiome following critical illness and the effect of probiotic or synbiotics on restoring a healthy microbiome in treated patients [66]. Recent advances in microbiome sequencing technology (16 s rRNA) in the last few years have resulted in an unprecedented growth in the amount of sequence data that can be collected at a previously unattainable low cost [66]. Thus, if we speculate that a specific probiotic or synbiotic therapy can be used to treat dysbiosis (a pathological change in the patient's bacterial flora) and restore a healthy microbiome, we need to evaluate this with the new accessible microbiome analysis techniques currently available. This may help us target probiotic or probiotic mixtures in the future and increase the personalization of care.

The strength of this current systematic review includes the use of several methods to reduce bias (comprehensive literature search, duplicate data abstraction, specific criteria for searching and analysis), and the analysis of relevant clinical outcomes in the critically ill. However, several important limitations in drawing strong treatment inferences are present. These include the significant potential for publication bias for the infection and hospital LOS outcomes, and the small numbers of trials included in subgroup analyses. In addition, the variety of probiotic strains, wide range of daily doses, and length of administration of probiotic therapy among the different trials weaken any possible clinical conclusions and recommendations. We were also unable to perform subgroup analysis for all clinical outcomes due to the limited number of studies evaluating each endpoint.

Based on our current data, there is not currently sufficient evidence to make a final strong recommendation for probiotics to be utilized in the prevention of infections, including VAP, in the critically ill. However, our current guideline recommendations suggest that probiotics should be considered to improve outcome in critically ill patients [19]. Future trials continue to need to address questions about timing, daily dose, and duration of therapy, which still remain unanswered.

\section{Conclusion}

In the largest systematic review and meta-analysis of probiotics to date, we demonstrated that in 30 trials enrolling 2972 patients, probiotics significantly reduced the incidence of infectious complications, including new episodes of VAP in critically ill patients. This finding is limited by clinical heterogeneity and potential publication bias for the overall infection outcome. This precludes a more meaningful statistical conclusion of the efficacy of probiotic therapy on overall infections and potentially the prevention of VAP in critical illness. Moreover, according to our findings probiotics has been demonstrated to be more effective in those trials with higher mortality in the control group. Probiotic therapy with $L$. plantarum currently demonstrates the most significant effect on the reduction of infections. Overall, the variety of strains, wide range of daily doses, and length of administration of probiotics weakens the strength of our conclusion. Certainly, additional large-scale, adequately powered, well-designed clinical trials, aimed at confirming our observations, are needed and warranted.

\section{Key messages}

- Critical illness is characterized by a loss of commensal flora and an overgrowth of potentially pathogenic bacteria, leading to a high susceptibility of nosocomial infections

- Probiotics are living non-pathogenic microorganisms, which may protect the gut barrier, attenuate pathogen overgrowth, decrease bacterial translocation and prevent infection in ICU patients

- Probiotic use in the ICU remains widespread and controversial, current guidelines are not conclusive, and a significant number of new trials of probiotics have been published recently, which requires a current and comprehensive systematic analysis of probiotic and synbiotic therapy in critically ill patients

- Probiotics were associated with a significant reduction in infections and a significant reduction in the incidence of ventilator-associated pneumonia (VAP) was found in critically ill patients receiving probiotics alone versus synbiotic mixtures, demonstrating the greatest improvement in infectious outcome, limited synbiotic trial data are currently available 
- Currently, clinical heterogeneity and potential publication bias reduce strong clinical recommendations and indicate further high-quality clinical trials are needed to conclusively prove these benefits

- Probiotics shows promise for the reduction of infections, including VAP in critical illness, and should be considered in critically ill patients

\section{Abbreviations}

CFU, colony-forming unit; Cl, confidence interval; C.Random, concealed randomization; EN, enteral nutrition; ICU, intensive care unit; Ig A, immunoglobulin $A$; ITT, intention to treat; $L G G$, Lactobacillus rhamnosus strain GG; LOS, length of stay; MV, mechanical ventilation; NA, non-attributable; NR, non-reported; OR, odds ratio; RCT, randomized controlled trial; RNA, ribonucleic acid; RR, risk ratio; VAP, ventilator-associated pneumonia; WMD, weighted mean difference

\section{Funding}

No funding for the development, writing or submission of this manuscript was received.

\section{Authors' contributions}

WM contributed to development of the concept of the manuscript, study grading, study selection, evaluation and interpretation of data, and also performed primary authoring and editing of all drafts of the manuscript. ML contributed to study grading, selection, evaluation and interpretation of data, performed much of the primary statistical analysis, meta-analysis and data analysis, and also contributed to the writing of the manuscript. $\mathrm{PL}$ contributed to development of study grading, study selection, evaluation and interpretation of data, and also contributed substantially to the writing of the manuscript. PW contributed to development of the concept of the manuscript, evaluation and interpretation of data, and also performed authoring and editing of all drafts of the manuscript. All authors read and approved the final manuscript.

\section{Competing interests}

The authors declare that they have no competing interests.

\section{Author details}

${ }^{1}$ Department of Critical Care, Intensive Care Unit, Hospital de Clínicas (University Hospital), Faculty of Medicine, Universidad de la República (UdelaR), Italia Av, 14th Floor, 11.600, Montevideo, Uruguay. ${ }^{2}$ Clinical Evaluation Research Unit. Angada 4, Kingston General Hospital, 76 Stuart Street, Kingston, ON K7L 2V7, Canada. ${ }^{3}$ Département de Anesthésie et de Réanimation, Faculté de Médecine et des Sciences de la Santé, Université de Sherbrooke, Centre Hospitalier Universitaire de Sherbrooke-Hôpital Fleurimont, Pièce 3610 3001, 12e Avenue Nord, Sherbrooke, QC J1H 5N4, Canada. ${ }^{4}$ Department of Anesthesiology and Pediatrics (Nutrition Section), University of Colorado, School of Medicine, 12700 E. 19th Ave., RC2 P15-7120, Box 8602, Aurora, CO 80045, USA.

Received: 28 April 2016 Accepted: 22 July 2016

Published online: 19 August 2016

\section{References}

1. Alverdy JC, Chang FB. The reemerging role of the intestinal flora in critical illness and inflammation: why the gut hypothesis of sepsis syndrome will not go away. J Leukoc Biol. 2008:83:461-6.

2. Latorre M, Krishnareddy S, Freedberg DE. Microbiome as mediator: Does systemic infection start in the gut? World J Gastroenterol. 2015;21:10487-92.

3. Watson RS, Carcillo JA, Linde-Zwirble WT, Clermont G, Lidicker J, Angus DC. The epidemiology of severe sepsis in children in the United States. Am J Respir Crit Care Med. 2003;167:695-701.

4. Milbrandt EB, Kersten A, Rahim MT, Dremsizov TT, Clermont G, Cooper LM, Angus DC, Linde-Zwirble WT. Growth of intensive care unit resource use and its estimated cost in Medicare. Crit Care Med. 2008:36:2504-10.

5. Food and Agricultural Organization of the United Nations and World Health Organization. Health and nutritional properties of probiotics in food including powder milk with live lactic acid bacteria. World Health Organization [online], http://who.int/foodsafety/fs_management/en/ probiotic_guidelines.pdf. 2001. Accessed 15 June 2016.

6. Luyer MD, Buurman WA, Hadfoune M, Speelmans G, Knol J, Jacobs JA, Dejong $\mathrm{CH}$, Vriesema AJ, Greve JW. Strain-specific effects of probiotics on gut barrier integrity following hemorrhagic shock. Infect Immun. 2005;73:3686-92.

7. Tok D, Ilkgul O, Bengmark S, Aydede H, Erhan Y, Taneli F, Ulman C, Vatansever S, Kose C, Ok G. Pretreatment with pro- and synbiotics reduces peritonitis-induced acute lung injury in rats. J Trauma. 2007;62:880-5.

8. Corr SC, Li Y, Riedel CU, O'Toole PW, Hill C, Gahan CG. Bacteriocin production as a mechanism for the antiinfective activity of Lactobacillus salivarius UCC118. Proc Natl Acad Sci U S A. 2007;104:7617-21.

9. Mittal R, Coopersmith CM. Redefining the gut as the motor of critical illness. Trends Mol Med. 2014;20(4):214-23.

10. Morrow LE, Kollef MH, Casale TB. Probiotic prophylaxis of ventilatorassociated pneumonia: a blinded, randomized, controlled trial. Am J Respir Crit Care Med. 2010;182:1058-64.

11. Hranjec T, Sawyer RG. Management of infections in critically ill patients. Surg Infect. 2014;15:474-8.

12. Siempos II, Ntaidou TK, Falagas ME. Impact of the administration of probiotics on the incidence of ventilator-associated pneumonia: a meta-analysis of randomized controlled trials. Crit Care Med. 2010;38:954-62.

13. Barraud D, Bollaert PE, Gibot S. Impact of the administration of probiotics on mortality in critically ill adult patients. Chest. 2013;143:646-55.

14. Petrof E, Dhaliwal R, Manzanares W, Johnstone J, Cook D, Heyland DK. Probiotics in the critically ill: a systematic review of the randomized trial evidence. Crit Care Med. 2012;40:3290-302.

15. Wang J, Liu KX, Ariani F, Tao LL, Zhang J, Qu JM. Probiotics for preventing ventilator-associated pneumonia: a systematic review and meta-analysis of high-quality randomized controlled trials. PLoS One. 2013;8, e83934.

16. Gu WJ, Wei CY, Yin RX. Lack of efficacy of probiotics in preventing ventilator-associated pneumonia: a systematic review and meta-analysis of randomized controlled trials. Chest. 2012;142:859-68.

17. Bo L, Li J, Tao T, Bai Y, Ye X, Hotchkiss RS, Kollef MH, Crooks NH, Deng X. Probiotics for preventing ventilator-associated pneumonia. Cochrane Database Syst Rev. 2014;(10)CD009066.

18. McClave SA, Taylor BE, Martindale RG, Warren MM, Johnson DR, Braunschweig C, McCarthy MS, Davanos E, Rice TW, Cresci GA, Gervasio JM, Sacks GS, Roberts PR, Compher C, Society of Critical Care Medicine; American Society for Parenteral and Enteral Nutrition. Guidelines for the Provision and Assessment of Nutrition Support Therapy in the Adult Critically III Patient: Society of Critical Care Medicine (SCCM) and American Society for Parenteral and Enteral Nutrition (A.S.P.E.N.). JPEN J Parenter Enteral Nutr. 2016:40:159-211.

19. Canadian Clinical Practice Guidelines. www.criticalcarenutrition.com Accessed March 2016.

20. Cui LH, Wang XH, Peng LH, Yu L, Yang YS. The effects of early enteral nutrition with addition of probiotics on the prognosis of patients suffering from severe acute pancreatitis. Zhonghua Wei Zhong Bing Ji Jiu Yi Xue. 2013;25:224-8.

21. Tan M, Lu X, Duan JW, Peng H, Zhu JC. Effects of probiotics on blood glucose levels and clinical outcomes in patients with severe cranocerebra trauma. Chin Crit Care Med. 2013;25:627-30.

22. Wang G, Wen J, Xu L, Zhou S, Gong M, Wen P, Xiao X. Effect of enteral nutrition and ecoimmunonutrition on bacterial translocation and cytokine production in patients with severe acute pancreatitis. J Surg Res. 2013;183:592-7.

23. López de Toro Martín-Consuegra I, Sanchez-Casado M, Pérez-Pedrero Sánchez-Belmonte MJ, López-Reina Torrijos P, Sánchez-Rodriguez P, Raigal-Caño A, Heredero-Galvez E, Zubigaray SB, Arrese-Cosculluela MÁ. The influence of symbiotics in multi-organ failure: randomised trial. Med Clin (Barc). 2014;143:143-9.

24. Sanaie S, Ebrahimi-Mameghani M, Hamishehkar H, Mojtahedzadeh M, Mahmoodpoor A. Effect of a multispecies probiotic on inflammatory markers in critically ill patients: A randomized, double-blind, placebocontrolled trial. J Res Med Sci. 2014;19:827-33.

25. Rongrungruang Y, Krajangwittaya D, Pholtawornkulchai K, Tiengrim S, Thamlikitkul V. Randomized controlled study of probiotics containing Lactobacillus casei (Shirota strain) for prevention of ventilator-associated pneumonia. J Med Assoc Thai. 2015;98:253-159. 
26. Zeng J, Wang CT, Zhang FS, Qi F, Wang SF, Ma S, Wu TJ, Tian H, Tian ZT, Zhang SL, Qu Y, Liu LY, Li YZ, Cui S, Zhao HL, Du QS, Ma Z, Li CH, Li Y, Si M, Chu YF, Meng M, Ren HS, Zhang JC, Jiang JJ, Ding M, Wang YP. Effect of probiotics on the incidence of ventilator-associated pneumonia in critically ill patients: a randomized controlled multicenter trial. Intensive Care Med. 2016. Epub ahead of print.

27. Wheeler KE, Cook DJ, Mehta S, Calce A, Guenette M, Perreault MM, Thiboutot Z, Duffett M, Burry L. Use of probiotics to prevent ventilator-associated pneumonia: A survey of pharmacists' attitudes. J Crit Care. 2016;31:221-6.

28. DerSimonian R, Laird N. Meta-analysis in clinical trials. Control Clin Trials. 1986;7:177-88.

29. Egger M, Davey Smith G, Schneider M, Minder C. Bias in meta-analysis detected by a simple, graphical test. BMJ. 1997;315:629-34

30. Tempe JD, Steidel AL, Blehaut H, Hasselmann M, Lutun P, Maurier F. Prevention of diarrhea administering Saccharomyces boulardii during continuous enteral feeding. Sem Hosp. 1983;59:1409-12.

31. Schlotterer $M$, Bernasconi $P$, Lebreton $F$, Wasserman D. Intérêt de Saccharomyces boulardii dans la tolérance digestive de la nutrition entérale à debit continu chez le brulé. Nutr Clin Métabol. 1987;1:31-4.

32. Heimburger DC, Sockwell DG, Geels WJ. Diarrhea with enteral feeding: prospective reappraisal of putative causes. Nutrition. 1994;10:392.

33. Bleichner $G$, Blehaut $H$, Mentec $H$, Moyse D. Saccharomyces boulardii prevents diarrhea in critically ill tube-fed patients. A multicenter, randomized, double-blind placebo-controlled trial. Intensive Care Med. 1997;23:517-23

34. Kecskés G, Belágyi T, Oláh A. Early jejunal nutrition with combined pre- and probiotics in acute pancreatitis-prospective, randomized, double-blind investigations [Article in Hungarian]. Magy Seb. 2003;56:3-8.

35. Lu X, Han CM, Yu JX, Fu SZ. Preliminary comparative study on the effects of early enteral supplementation of synbiotics on severely burned patients. [Article in Chinese]. Zhonghua Shao Shang Za Zhi. 2004;20:198-201.

36. Jain PK, McNaught CE, Anderson AD, MacFie J, Mitchell CJ. Influence of synbiotic containing Lactobacillus acidophilus La5, Bifidobacterium lactis Bb 12, Streptococcus thermophilus, Lactobacillus bulgaricus and oligofructose on gut barrier function and sepsis in critically ill patients: a randomised controlled trial. Clin Nutr. 2004;23:467-75.

37. Klarin B, Johansson ML, Molin G, Larsson A, Jeppsson B. Adhesion of the probiotic bacterium Lactobacillus plantarum 299v onto the gut mucosa in critically ill patients: a randomised open trial. Crit Care. 2005;9:R285-293.

38. McNaught CE, Woodcock NP, Anderson AD, MacFie J. A prospective randomised trial of probiotics in critically ill patients. Clin Nutr. 2005:24:211-9.

39. Kotzampassi K, Giamarellos-Bourboulis EJ, Voudouris A, Kazamias P, Eleftheriadis E. Benefits of a symbiotic formula (Synbiotic 2000Forte) in critically ill trauma patients: early results of a randomized controlled trial. World J Surg. 2006;30:1848-55.

40. Alberda C, Gramlich L, Meddings J, Field C, McCargar L, Kutsogiannis D, Fedorak R, Madsen K. Effects of probiotic therapy in critically ill patients: a randomized, double-blind, placebo-controlled trial. Am J Clin Nutr. 2007;85:816-23.

41. Li YM. Adjuvant therapy for probiotics in patients with severe acute pancreatitis: an analysis of 14 cases. World Chin J Digestol. 2007;15:302-4.

42. Olah A, Belagyi T, Poto L, Romics Jr L, Bengmark S. Synbiotic control of inflammation and infection in severe acute pancreatitis: a prospective, randomized, double blind study. Hepatogastroenterolgy. 2007:54:590-4.

43. Besselink MG, van Santvoort HC, Buskens E, Boermeester MA, van Goor $H$, Timmerman HM, Nieuwenhuijs VB, Bollen TL, van Ramshorst B, Witteman BJ, Rosman C, Ploeg RJ, Brink MA, Schaapherder AF, Dejong $\mathrm{CH}$, Wahab PJ, van Laarhoven CJ, van der Harst E, van Eijck CH, Cuesta MA, Akkermans LM, Gooszen HG, Dutch Acute Pancreatitis Study Group. Probiotic prophylaxis in predicted severe acute pancreatitis: a randomised, double-blind, placebo-controlled trial. Lancet. 2008;371:651-9.

44. Forestier C, Guelon D, Cluytens V, Gillart T, Sirot J, De Champs C. Oral probiotic and prevention of Pseudomonas aeruginosa infections: a randomized, double-blind, placebo-controlled pilot study in intensive care unit patients. Crit Care. 2008;12:R69.

45. Klarin B, Wullt M, Palmquist I, Molin G, Larsson A, Jeppsson B. Lactobacillus plantarum 299v reduces colonisation of Clostridium difficile in critically ill patients treated with antibiotics. Acta Anaesthesiol Scand. 2008;52:1096-102
46. Knight DJ, Gardiner D, Banks A, Snape SE, Weston VC, Bengmark S, Girling $\mathrm{KJ}$. Effect of synbiotic therapy on the incidence of ventilator associated pneumonia in critically ill patients: a randomised, double-blind, placebocontrolled trial. Intensive Care Med. 2009:35:854-61.

47. Barraud D, Blard C, Hein F, Marçon O, Cravoisy A, Nace L, Alla F, Bollaert PE, Gibot S. Probiotics in the critically ill patient: a double blind, randomized, placebo-controlled trial. Intensive Care Med. 2010;36:1540-7.

48. Frohmader TJ, Chaboyer WP, Robertson IK, Gowardman J. Decrease in frequency of liquid stool in enterally fed critically ill patients given the multispecies probiotic VSL\#3: a pilot trial. Am J Crit Care. 2010;19:e1-11.

49. Ferrie S, Daley M. Lactobacillus GG as treatment for diarrhea during enteral feeding in critical illness: randomized controlled trial. JPEN J Parenter Enteral Nutr. 2011;35:43-9.

50. Sharma B, Srivastava S, Singh N, Sachdev V, Kapur S, Saraya A. Role of probiotics on gut permeability and endotoxemia in patients with acute pancreatitis: a double-blind randomized controlled trial. J Clin Gastroenterol. 2011:45:442-8

51. Tan M, Zhu JC, Du J, Zhang LM, Yin HH. Effects of probiotics on serum levels of Th1/Th2 cytokine and clinical outcomes in severe traumatic brain-injured patients: a prospective randomized pilot study. Crit Care. 2011;15:R290.

52. Morrow LE, Gogineni V, Malesker MA. Synbiotics and probiotics in the critically ill after the PROPATRIA trial. Curr Opin Clin Nutr Metab Care. 2012;15:147-50.

53. Sheldon T. Dutch probiotics study is criticised for its "design, approval, and conduct". BMJ. 2010:340:c77.

54. Gou S, Yang Z, Liu T, Wu H, Wang C. Use of probiotics in the treatment of severe acute pancreatitis: a systematic review and meta-analysis of randomized controlled trials. Crit Care. 2014;18:R57.

55. Hempel S, Newberry S, Ruelaz A, Wang Z, Miles JNV, Suttorp MJ, Johnsen B, Shanman R, Slusser W, Fu N, Smith A, Roth E, Polak J, Motala A, Perry T, Shekelle PG. Safety of Probiotics to Reduce Risk and Prevent or Treat Disease. Evidence Report/Technology Assessment No. 200. (Prepared by the Southern California Evidence-based Practice Center under Contract No. 2902007-10062-I.) AHRQ Publication No. 11-E007. April 2011. Available at: www. ahrq.gov/clinic/tp/probiotictp.htm. Accessed 15 June 2016.

56. De Groote MA, Frank DN, Dowell E, Glode MP, Pace NR. Lactobacillus rhamnosus GG bacteremia associated with probiotic use in a child with short gut syndrome. Pediatr Infect Dis J. 2005;24:278-80.

57. Vincent JL, Rello J, Marshall J, Silva E, Anzueto A, Martin CD, Moreno R, Lipman J, Gomersall C. International study of the prevalence and outcomes of infection in intensive care units. JAMA. 2009;302:2323-9.

58. Ego A, Preiser JC, Vincent JL. Impact of diagnostic criteria on the incidence of ventilator-associated pneumonia. Chest. 2015;147:347-55.

59. Kollef MH, Shorr A, Tabak YP, Gupta V, Liu LZ, Johannes RS. Epidemiology and outcomes of health-care-associated pneumonia: results from a large US database of culture positive pneumonia. Chest. 2005;128:3854-62.

60. Rello J, Ollendorf DA, Oster G, Vera-Llonch M, Bellm L, Redman R, Kollef $\mathrm{MH}$, VAP Outcomes Scientific Advisory Group. Epidemiology and outcomes of ventilator-associated pneumonia in a large US database. Chest. 2002;122:2115-21.

61. Branch-Elliman W, Wright SB, Howell MD. Determining the ideal strategy for ventilator-associated pneumonia. Am J Respir Crit Care Med. 2015:192:57-63.

62. Hempel S, Newberry SJ, Maher AR, Wang Z, Miles JN, Shanman R, Johnsen $B$, Shekelle PG. Probiotics for the prevention and treatment of antibiotic-associated diarrhea: a systematic review and meta-analysis. JAMA. 2012;307:1959-69.

63. Goldenberg JZ, Ma SS, Saxton JD, Martzen MR, Vandvik PO, Thorlund K, Guyatt GH, Johnston BC. Probiotics for the prevention of Clostridium difficile-associated diarrhea in adults and children. Cochrane Database Syst Rev. 2013;5, CD006095

64. Glenting J, Beck HC, Vrang A, Riemann H, Ravn P, Hansen AM, Antonsson M, Ahrné S, Israelsen H, Madsen S. Anchorless surface associated glycolytic enzymes from Lactobacillus plantarum299v bind to epithelial cells and extracellular matrix proteins. Microbiol Res. 2013;168:245-53.

65. Gross G, Snel J, Boekhorst J, Smits MA, Kleerebezem M. Biodiversity of mannosespecific adhesion in Lactobacillus plantarum revisited: strain-specific domain composition of the mannose-adhesin. Benef Microbes. 2010;1:61-6.

66. Ursell LK, Metcalf JL, Parfrey LW, Knight R. Defining the human microbiome. Nutr Rev. 2012;70 Suppl 1:S38-44. 